NASACONTRACTOR $R E P O R T$

$$
\begin{aligned}
& m \\
& 0 \\
& \sim \\
& 0 \\
& \cup \\
& \approx \\
& z
\end{aligned}
$$

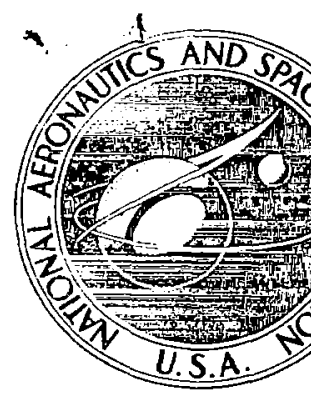

\title{
HIGHER-ORDER NUMERICAL SOLUTIONS USING CUBIC SPLINES
}

S. G. Rubin and P. K. Kbosla

Prepared by

POLYTECHNIC INSTITUTE OF NEW YORK

Farmingdale, N. Y. 11735

for Langley Research Center

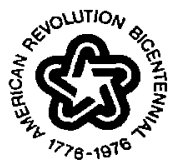

NATIONAI. AERONAUTICS AND SPACE ADMINISTRATION • WASHINGTON, D. C. • FEBRUARY 1976 


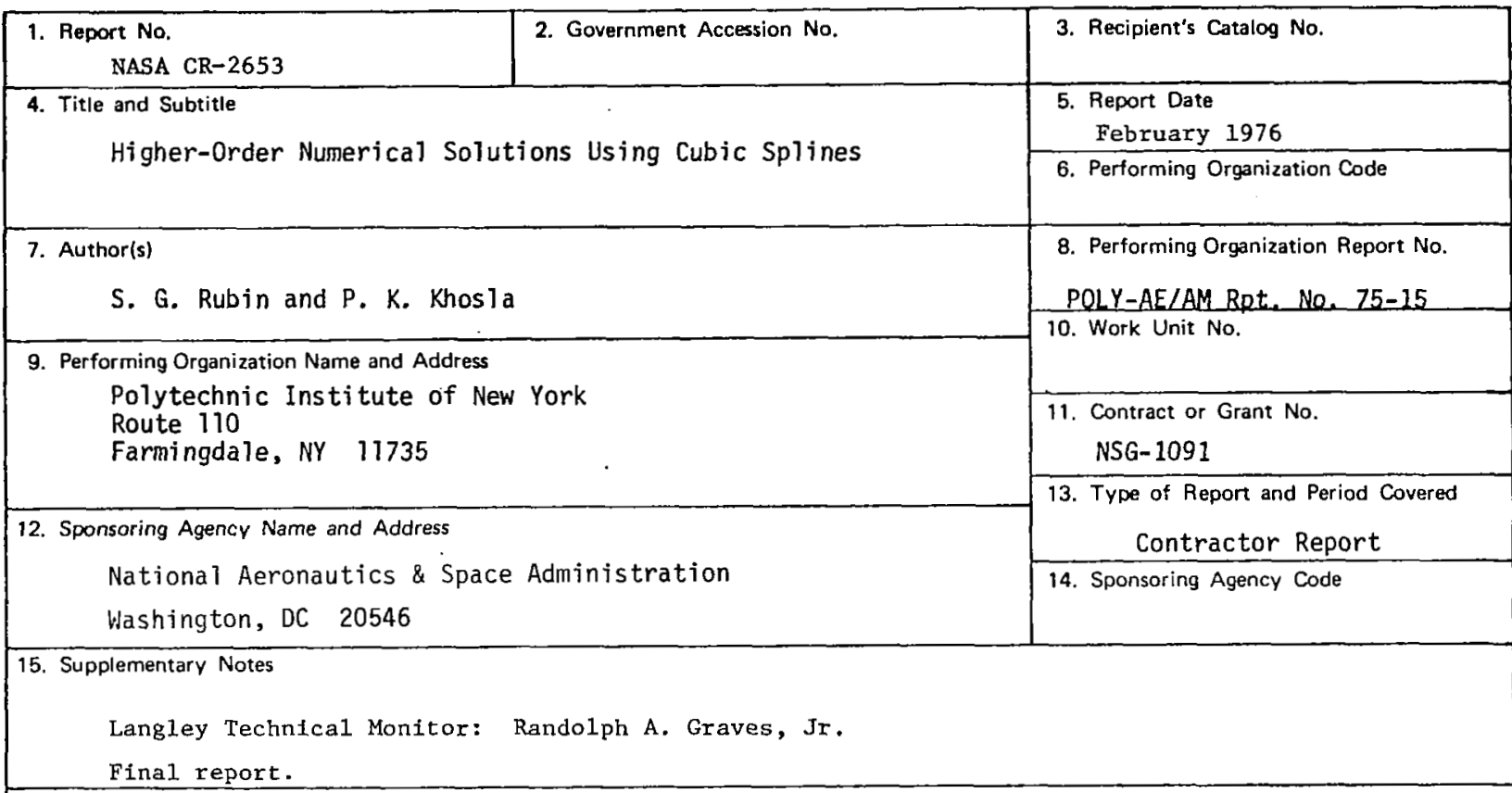

16. Abstract

A cubic spline collocation procedure has recently been developed for the numerical solution of partial differential equations. In the present paper, this spline procedure is reformulated so that the accuracy of the second-derivative approximation is improved and parallels that previously obtained for lower derivative terms. The final result is a numerical procedure having overall third-order accuracy for a non-uniform mesh. Solutions using both spline procedures, as well as three-point finite difference methods, will be presented for several model problems.

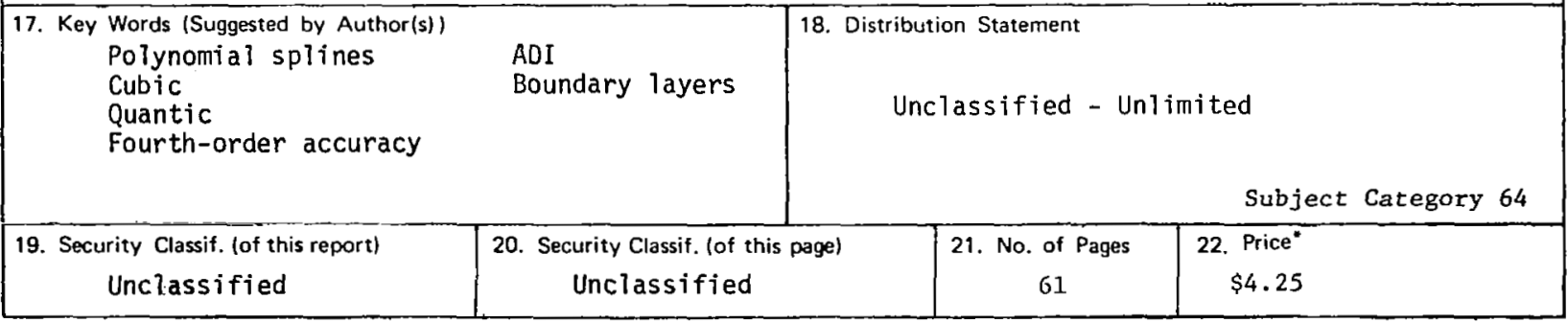


TABLE OF CONTENTS

Section

Page

Abstract 1

I Introduction 1

II Spline 2 - Review of Cubic Spline Theory 6

III Spline 4-Derivation and Discussion 12

IV Finite-Difference Theory/Spline $1 \quad 14$

$\begin{array}{ll}\text { V Stability } & 15\end{array}$

$\begin{array}{llr}\text { VI } & \text { Results } & 18\end{array}$

A. Burgers Equation 19

B. Iinear Burgers Equation 22

C. Linear Corner Flow 25

D. Laplace Equation 27

E. Potential Flow Over a Circular Cylinder 30

F. Similarity Boundary Layers 31

G. Non-Similar Boundary Layer Analysis 34

$\begin{array}{lll}\text { VII Summary } & 35\end{array}$

References $\quad 36$ 


\section{LIST OF TABLES}

Table

Page

1 Solution of Burgers Equation $v=1 / 8, \sigma=1.0,31$ Equally spaced Points

2 Solution of Burgers Equation $\nu=1 / 8, \sigma=1.2,15$

Points

3 Solution of Burgers Equation: $\nu=1 / 8, \sigma=1.8$,

15 Points

4 Solution of Burgers Equation: $\nu=1 / 16, \sigma=1.0$,

19 Equaliy Spaced Points

5 Solution of Burgers Equation: $\nu=1 / 24, \sigma=1.2$,

31 Points

6 Linear Burgers Equation

7 Linearized Corner Flow

8 Solution of the Laplace Equation

9 Potential Flow over a Circular Cylinder

10 Potential Flow over a circular Cylinder

I1 Slip Velocity on the front of a circular Cylinder, $\Delta \theta=\pi / 10$

12 Blasius Profile: $\sigma=1.0, h_{2}=0.1, N=61$

13 Blasius Profile: $\sigma=1.0, h_{2}=1.0, N=21$

14 Blasius Profile: $\sigma=1.8 / 1, h_{2}=0.5, N=21$ 


\section{LIST OF FIGURES}

Figure

Page

1 Non-Iinear Burgers Equation: $\nu=1 / 8, \sigma=1$

2 Laplace Equation

3 Error Plot: Blasius Equation

4 Constant Pressure Boundary Layer Solution -

Physical Variables 
HIGHER-ORDER NUMERICAL SOLUTIONS USING CUBIC SPIINES

\author{
S. G. Rubin and P. K. Khosla \\ Polytechnic Institute of New York \\ Farmingdale, New York
}

\begin{abstract}
A cubic spline collocation procedure has recently been developed for the numerical solution of partial differential equations. In the present paper, this spline procedure is reformulated so that the accuracy of the second-derivative approximation is improved and parallels that previously obtained for lower derivative terms. The final result is a numerical procedure having overall third-order accuracy for a non-uniform mesh and overall fourth-order accuracy for a uniform mesh. Solutions using both spline procedures, as well as three-point finite difference methods, will be presented for several model problems.
\end{abstract}

\title{
I. INTRODUCTION
}

In a recent study Rubin and Graves $^{1,2}$ have presented a cubic spline 3,4 collocation procedure for the numerical solution of partial differential equations. This technique exhibits the following desirable features: (I) The governing matrix system is always tridiagonal so that well-developed and highly efficient inversion algorithms are applicable; (2) cubic spline interpolation leads to second order accuracy 
for second derivatives, e.g., diffusion terms in the NavierStokes equations. This order of accuracy is maintained even with rather large non-uniformities in mesh width; (3) first derivatives or convection effects are fourth-order accurate for a uniform mesh and third-order with mesh non-uniformity; (4) derivative boundary conditions can in many cases be applied more accurately and with less difficulty than with conventional finite-difference schemes; (5) a simple two-point relationship exists between the spline approximation for the first and second derivatives; and (6) unlike finite-element or other Galerkin (integral) methods, which are generally not tridiagonal, the evaluation of large numbers of quadratures is unnecessary.

Solutions have been obtained for a number of problems ${ }^{1,2}$ with explicit, implicit and spline alternating direction implicit (SADI) temporal or spatial marching procedures. Moreover, for the viscous and potential flow problems considered, it was found that with the spline procedure there was no particular advantage gained with the equations in divergence form. In some recent studies it has been found that the divergence form may be desirable with flux boundary conditions. These results are described later in this paper. Agreement of the spline solutions with exact analytic results and very accurate finite-difference solutions obtained with a very fine mesh has been quite good ${ }^{1,2}$. All comparisons ${ }^{1,2}$ with conventional three-point finite difference formulations 
demonstrate the improved spline accuracy associated with (i) the higher-order convection approximation, (ii) the treatment of derivative boundary conditions, or (iii) the higherorder accuracy of spline second derivatives (diffusion) when specifying a non-uniform mesh. Solutions for the Burgers equation, the two-dimensional diffusion equation and the incompressible viscous flow in a driven cavity are found in Refs. 1 and 2 .

In the present paper, the cubic spline procedure is reformulated so that the accuracy of the second-derivative approximation is improved and parallels that obtained for the lower derivative terms. The final result is a combined splinefinite difference numerical procedure having overall thirdorder spatial accuracy for non-uniform meshes and overall fourth-order spatial accuracy with a uniform mesh. In order to differentiate the two spline procedures, we shall designate the original spline formulation ${ }^{1,2}$ as spline 2 and the improved formulation presented here as spline 4.

As shown in sections II and III, the cubic spline collocation procedure involves a third-order interpolation polynomial with the function and the second (or first) derivative of the function as unknowns at each mesh point. Continuity of the first (or second) derivative leads to the tridiagonal system of equations to be considered. In section IV, it is shown how the familiar central difference second-order accurate finitedifference theory results from a quadratic spline interpolation procedure. Using the earlier spline designation, the finite- 
difference theory is classified as spline 1.

Recently, several higher-order finite-difference schemes with similar properties have been proposed; i.e., the functions and derivatives are considered unknown at each mesh point, or the functions are collocated at three points instead of one. The methods which have been termed Hermitian finite-difference 5,6 Padé approximation ${ }^{7}$ or compact differencing ${ }^{8}$, and Mehrstellung 9 have been developed for a uniform mesh and have somewhat lower truncation errors than the five-point pentadiagonal fourth-order finite difference procedure. As with the spline formulation, they remain of tridiagonal form.

The authors have examined these procedures, as well as a fourth-order spline-on-spline method, and found them to be, in fact, identical; i.e., any one can be derived from any of the others. As with spline 4, these finite-difference or splineon methods are fourth-order with a uniform mesh and thirdorder with a non-uniform mesh. The main differences are handling of the boundary conditions, the relationship between the approximations for the convection and diffusion terms, and the truncation errors. The truncation errors for first derivatives are identical. The truncation error for the secondderivative to be discussed later for spline 4 is $50 \%$ smaller than that found with the higher-order finite-difference or 
spline-on-spline collocation formulae.

In order to evaluate the spline procedures, the truncation errors, stability limitations and effects of boundary conditions will be discussed. Spline 2 is reviewed in section II, spline 4 is introduced and discussed in section III, and spline 1 is presented in section IV. The stability conditions for all methods are outlined in section $v$. Solutions using both spline procedures, as well as a three-point finite-difference method, are presented for several model problems in section VI. Both uniform and non-uniform meshes are considered. In each case the analytic solution or a very accurate numerical solution is available for comparison purposes. The problems to be considered include (I) a boundary layer-like solution of Laplace's equation, where a spline relaxation method is applied, (2) potential flow over a circular cylinder with a spline successive approximation procedure, (3) the weak shock solution for the nonlinear Burgers equation by a two-step explicit or an implicit spline integration, (4) divergence and non-divergence solutions for the linear Burgers equation with flux and other derivative boundary conditions, (5) the impulsive motion of right angle corner (Rayleigh problem) with SADI, (6) the solution of the two-point boundary value problem describing similar boundary layer behavior, and (7) non-similar constant pressure boundary layer solutions for large Reynolds number using physical variables. The results will be summarized in section VII. 


\section{SPIINE 2 - REVIEW OF CUBIC SPLINE THEORY}

Consider a mesh with nodal points such that

$$
a=x_{0}<x_{1}<x_{2} \ldots<x_{N}<x_{N+1}=b
$$

and with

$$
h_{i}=x_{i}-x_{i-1}>0
$$

Consider a function $u(x)$ such that at the mesh points $x_{i}$ $u\left(x_{i}\right)=u_{i}$. The cubic spline is a function $s_{\Delta}\left(u_{i}, x\right)=s_{\Delta}(x)$ which is continuous together with its first and second derivatives on the interval $[a, b]$, corresponds to a cubic polynomial in each sub-interval $x_{i-1} \leq x \leq x_{i}$, and satisfies $s_{\Delta}\left(u_{i} ; x_{i}\right)=u_{i}$. In the usual spline terminology, spline 2 is defined as a cubic spline of deficiency one, since all but one of the three polynomial derivatives are continuous.

If $u(x)$ and its derivatives are continuous, it has been shown that the spline function $S_{\Delta}(x)$ approximates $u(x)$ at all: points in $[a, b]$ to fourth order in $\max h_{i}$. First and second derivatives of $S_{\Delta}(x)$ approximate $u^{\prime}(x)$ and $u^{\prime \prime}(x)$ to third and second order, respectively. See Ahlberg, Nilson and Walsh ${ }^{3}$ for detailed proofs of convergence.

If $S_{\Delta}(x)$ is cubic on $\left[x_{i-1}, x_{i}\right]$, then in general,

$$
S_{\Delta}^{\prime \prime}(x)=M_{i-1}\left(\frac{x_{i}-x}{h_{i}}\right)+M_{i}\left(\frac{x-x_{i-1}}{h_{i}}\right),
$$

where $M_{i} \equiv S_{\Delta}^{\prime \prime}\left(x_{i}\right)$

Integrating twice leads to the interpolation formula on $\left[x_{i-1}, x_{i}\right]$ 


$$
\begin{aligned}
& S_{\Delta}(x)=M_{i-1} \frac{\left(x_{i}-x\right)^{3}}{6 h_{i}}+M_{i} \frac{\left(x-x_{i-1}\right)^{3}}{6 h_{i}} \\
& \quad\left(u_{i-1}-\frac{M_{i-1} h_{i}^{a}}{6}\right) \frac{\left(x_{i}-x\right)}{h_{i}}+\left(u_{i}-\frac{M_{i} h_{i}^{a}}{6}\right) \frac{\left(x-x_{i-1}\right)}{h_{i}} .
\end{aligned}
$$

The constants of integration have been evaluated from $S_{\Delta}\left(x_{i}\right)=u_{i}$ and $s_{\Delta}\left(x_{i-1}\right)=u_{i-1} \cdot s_{\Delta}(x)$ on $\left[x_{i}, x_{i+1}\right]$ is obtained with $i+1$ replacing $i$ in (la).

The unknown derivatives $M_{i}$ are related by enforcing the continuity condition on $S_{\Delta}^{\prime}(x)$. With $S_{\Delta}^{\prime}\left(x_{i}^{-}\right)=m_{i}^{-}$on $[i-1, i]$ and $\mathrm{S}_{\Delta}^{\prime}\left(\mathrm{x}_{i}^{+}\right)=\mathrm{m}_{i}^{+}$on $\left[\mathrm{x}_{i}, \mathrm{x}_{i+1}\right]$, we require $\mathrm{m}_{i}^{-}=\mathrm{m}_{i}^{+}=\mathrm{m}_{i}$. We find for $i=1, \ldots, N$,

$$
\frac{h_{i}}{6} M_{i-1}+\frac{h_{i}+h_{i+1}}{3} M_{i}+\frac{h_{i+1}}{6} M_{i+1}=\frac{\left(u_{i+1}-u_{i}\right)}{h_{i+1}}-\frac{\left(u_{i}-u_{i-1}\right)}{h_{i}} .
$$

Additional spline relationships that are easily derived are listed below:

$$
\begin{aligned}
& \frac{1}{h_{i}} m_{i-1}+2\left(\frac{1}{h_{i}}+\frac{1}{h_{i+1}}\right) m_{i}+\frac{1}{h_{i+1}} m_{i+1}=\frac{3\left(u_{i+1}-u_{i}\right)}{h_{i+1}^{2}}+\frac{3\left(u_{i}-u_{i-1}\right)}{h_{i}^{2}} ; \\
& m_{i+1}-m_{i}=\frac{h_{i+1}}{2}\left(M_{i}+M_{i+1}\right) ; \\
& m_{i}=\frac{h_{i}}{3} M_{i}+\frac{h_{i}}{6} M_{i-1}+\frac{u_{i}-u_{i-1}}{h_{i}} ; \\
& m_{i}=-\frac{h_{i+1}}{3} M_{i}-\frac{h_{i+1}}{6} M_{i+1}+\frac{u_{i+1}-u_{i}}{h_{i+1}} ;
\end{aligned}
$$




$$
\begin{aligned}
& M_{i}=\frac{2 m_{i-1}}{h_{i}}+\frac{4 m_{i}}{h_{i}}-6 \frac{u_{i}-u_{i-1}}{h_{i}^{2}} ; \\
& M_{i}=-\frac{4 m_{i}}{h_{i+1}}-\frac{2 m_{i+1}}{h_{i+1}}+6 \frac{u_{i+1}-u_{i}}{h_{i+1}^{2}} .
\end{aligned}
$$

Eqs. (IB) or (IC) lead to a system of $N$ equations for the $N+2$ unknowns $\mathrm{M}_{i}$ or $\mathrm{m}_{i}$, respectively. The additional two equations are obtained from boundary conditions on $m_{0}, m_{N+1}$ or $M_{0}, M_{N+1}$ The resulting tridiagonal system for $M_{i}$ or $m_{i}$ is diagonally dominant and solved by an efficient inversion algorithm ${ }^{3}$. Spline 2 for Solving Partial Differential Equations ${ }^{1,2}$

If the values $u_{i}$ are not prescribed but represent the solution of a quasi-linear second order partial differential equation, $u_{t}=f\left(u, u_{x}, u_{x x}\right)$, then an approximate solution for $u_{i}$ can be obtained by considering the solution of

$$
\left(u_{t}\right)_{i}=f\left(u_{i}, m_{i}, M_{i}\right) \text {. }
$$

This formulation is designated spline 2. If the time derivative is discretized in a simple finite-difference fashion, we have

$$
\frac{u_{i}^{n+1}-u_{i}^{n}}{\Delta t}=(1-\theta) f^{n}+\theta f^{n+1} ;
$$

$\theta=0$, explicit; $\theta=1$, implicit; $\theta=\frac{1}{2}$, Crank-Nicolson. For the explicit integration the stability limitations are quite severe, see Refs. 1,2 and section VI. Therefore a two-step procedure is considered and is given as: 


$$
\begin{aligned}
& \text { Step 1: } \frac{\bar{u}_{i}^{n+1}-u_{i}^{n}}{\Delta t}=f^{n} \\
& \text { Step 2: } \frac{u_{i}^{n+1}-u_{i}^{n}}{\Delta t}=\bar{t}^{n+1}
\end{aligned}
$$

\section{Example:}

Consider the linear Burgers equation

$$
u_{t}+\bar{u} u_{x}=\nu u_{x x} ; \bar{u}=\bar{u}(x, t) ; \nu=\nu(x, t) \text {. }
$$

With (1b) and (1c) we obtain a system of $3 \mathrm{~N}$ equations for 3. $(\mathrm{N}+2)$ unknowns (see Refs. 1,2 for further details on the derivation). The system (2) can be written ${ }^{\dagger}$ as

$$
A_{i} v_{i-1}^{n+1}+B_{i} v_{i}^{n+1}+C_{i} v_{i+1}^{n+1}=D_{i} v_{i}^{n}+E_{i}\left[\sigma v_{i-1}^{n}+v_{i+1}^{n}\right]
$$

where

$$
\begin{aligned}
& A_{i}=\left[\begin{array}{ccc}
0 & 0 & \gamma_{I} \\
-1 / h_{i} & 0 & h_{i} / 6 \\
3 / h_{i}^{3} & 1 / h_{i} & 0
\end{array}\right] ; \\
& B_{i}=\left[\begin{array}{ccc}
(1+1 / \sigma) / h_{i} & \alpha_{1} & \alpha_{2} \\
-3\left(1-1 / \sigma^{2}\right) / h_{i}^{2} & 2(1+1 / \sigma) / h_{i} & 0
\end{array}\right] ; \\
& C_{i}=\left[\begin{array}{ccc}
0 & 0 & \gamma_{2} \\
-\frac{1}{h_{i+1}} & 0 & \frac{h_{i+1}}{6} \\
\frac{-3}{h_{i+1}^{2}} & \frac{1}{h_{i+1}} & 0
\end{array}\right] ;
\end{aligned}
$$

*It is possible to treat the viscous terms $\left(M_{i}\right)$ implicitly $(\theta=1)$ and the convection terms explicitly. As shown in Refs. 1, 2, the stability of the two-step procedure for viscous flows is improved. $t_{A}$ number of variations on this system can be derived with the relations (1). 


$$
\begin{aligned}
& D_{i}=\left[\begin{array}{lll}
\rho_{0} & \rho_{I} & \rho_{z} \\
0 & 0 & 0 \\
0 & 0 & 0
\end{array}\right] ; \\
& E_{i}=\left[\begin{array}{lll}
0 & 0 & \delta_{I} \\
0 & 0 & 0 \\
0 & 0 & 0
\end{array}\right] \\
& v_{i}=\left[\begin{array}{lll}
u_{i}, & m_{i} & M_{i}
\end{array}\right]^{T},
\end{aligned}
$$

and

$$
\begin{aligned}
& \sigma=h_{i+1} / h_{i} ; \gamma_{1}=\gamma_{2}=\delta_{1}=0 \\
& \alpha_{0}=1 ; \alpha_{1}=\theta \bar{u}_{i}^{n+1} \Delta t ; \alpha_{2}=-\theta \nu_{i}^{n+1} \Delta t ; \\
& \rho_{0}=1 ; \rho_{1} \equiv-(1-\theta) \bar{u}_{i}^{n} \Delta t ; \rho_{2}=(1-\theta) \nu_{i}^{n} \Delta t .
\end{aligned}
$$

A significant advantage of the spline 2 formulation is that with expressions (1) it is possible to reduce the $3 \times 3$ matrix system (3) to a scalar set of equations for $\mathrm{M}_{j}$ alone. The details of this reduction process are found in Refs. 1,2.

For equations with two space dimensions such that $u_{t}=f\left(u, u_{x}\right.$ $\left.u_{y}, u_{x x}, u_{y y}\right), a$ spline alternating direction implicit (SADI) procedure has been presented by Rubin and Graves ${ }^{1,2}$. A spline successive approximation method can also be simply formulated. Both techniques are discussed later in this paper where several example problems are presented. Truncation Error

For interior points, the spatial accuracy of the spline approximation can be directly estimated from the formulas (1b) 
and (le) or (If). Expanding $m_{i}, M_{i}$ and $u_{i}$ in Taylor series and assuming the necessary continuity of derivatives for $u(x, y)$, we obtain, with $\sigma=h_{i+1} / h_{i}$,

$$
\begin{aligned}
& \left(u_{x x}\right)_{i}=M_{i}+\left(u^{i v}\right)_{i} h_{i}^{2}\left(\sigma^{3}+1\right) / 12(\sigma+1) \\
& -\left(u^{v}\right)_{i} h_{i}^{3}(\sigma-1)\left(2 \sigma^{2}+5 \sigma+2\right) / 180 \\
& -\left(u^{v i}\right)_{i} h_{i}^{4}\left[\sigma^{2} / 360+(\sigma-1)^{2}\left(7 \sigma^{2}-2 \sigma+7\right) / 1080\right] \\
& +o\left(h_{i}^{5}\right) .
\end{aligned}
$$

and

$$
\begin{aligned}
& \left(u_{x}\right)_{i}=m_{i}+\left(u^{i v}\right)_{i} h_{i}^{3} \sigma(\sigma-1) / 24+ \\
& \quad+\left(u^{v}\right)_{i} h_{i}^{4} \sigma[1+\sigma(\sigma-1)] / 180+o\left(h_{i}^{5}\right) .
\end{aligned}
$$

Fyfe $e^{10}$ has presented similar relations,

for constant $h_{i}$, in his collocation analysis of cubic splines for the solution of two point boundary value problems.

Therefore, the spline approximation with a non-uniform mesh is second-order accurate for $M_{i}$ and third-order for $m_{i}$. For $a$ uniform mesh $m_{i}$ becomes fourth-order with $M_{i}$ remaining secondorder accurate. In the next section a finite-difference expression for $\left(u^{i v}\right)_{i}$ is used to increase the accuracy of $M_{i}$ and hence the overall accuracy of the procedure. With this modification this formulation will be termed spline 4 .

\footnotetext{
TIf (lc) is used to evaluate the truncation error for $m_{i}$, the constant 24 in the second expression on the right-hand side becomes 72. For the uniform case, (4b) is recovered in all cases.
} 


\section{SPLINE 4 - DERIVATION AND DISCUSSION}

In order to improve the overall accuracy of the spline 2 formulation, it is necessary to reduce the order of the truncation error for $\left(u_{x x}\right)_{i}$ in (4a). Although a number of procedures are possible, we have chosen a very simple modification, whereby the error term in (4a) for $\left(u^{i v}\right)_{i}$ is approximated by a threepoint discretization for $M_{i}$. This approximation is first-order accurate with a non-uniform mesh and second-order with a uniform mesh. Therefore the spline approximation for $\left(u_{x x}\right)_{i}$ is improved, and parallels that for $\left(u_{x}\right)_{i} ; i . e .$, third-order accuracy is achieved for a non-uniform grid and fourth-order accuracy for uniform mesh. This improvement leads to what is termed spline 4, or a quintic spline of deficiency three.

The development of spline 4 is as follows: The expression (4a) can be rewritten in the form

$$
\left(u_{x x}\right)_{i}=M_{i}+h_{i}^{2} \sigma(\sigma+1) \Delta\left(M_{x x^{\prime}}\right)_{i} / 12+o\left((\sigma-1) h_{i}^{3}, h_{i}^{4}\right)
$$

where $\Delta=\left(1+\sigma^{3}\right) / \sigma(1+\sigma)^{2}$.

The familiar three-point discretization formula is

$$
\begin{aligned}
& \left(M_{x x}\right)_{i}=\frac{2}{\sigma(\sigma+1) h_{i}^{2}}\left[M_{i+1}-(1+\sigma) M_{i}+\sigma M_{i-1}\right] \\
& -(\sigma-1) h_{i}\left(M_{x x x}\right)_{i} / 3-h_{i}^{3}\left(I+\sigma^{3}\right)\left(M^{i v}\right)_{i} / 12(1+\sigma)+O\left(h_{i}^{3}\right) .
\end{aligned}
$$


Therefore, (4a) or (5a) becomes

$$
\begin{aligned}
& \left(u_{x x}\right)_{i}=M_{i}+(\Delta / 6)\left(M_{i+1}-(1+\sigma) M_{i}+\sigma M_{i-1}\right) \\
& -7 h_{i}^{3}\left(1+\sigma^{2}\right)(\sigma-1)\left(u^{v}\right)_{i} / 180-h_{i}^{4}\left(u^{v i}\right)_{i}\left[\sigma^{2} / 360\right. \\
& \left.\quad+(\sigma-1)^{2}\left(7 \sigma^{2}-2 \sigma+7\right) / 1080\right]+o\left(h_{i}^{5}\right) .
\end{aligned}
$$

With (4b),

$$
\left(u_{x}\right)_{i}=m_{i}+O\left((\sigma-1) h_{i}^{3}, h_{i}^{4}\right)+
$$

and we obtain a uniform higher-order approximation termed spline $4 .^{\neq}$When $\sigma=1$, spline 4 is fourth-order accurate and the truncation error of (5b) is smaller than that obtained with Hermitian or Padé methods ${ }^{5-9}$, which are in turn smaller than the error obtained witn five-point finite-different discretizations.

In the spline 4 procedure the relations (lb-lh) still apply; however, the interpolation polynomial is no longer applicable as spline 4 represents a higher-order interpolation. The governing system

\footnotetext{
It is possible to apply $(5 \mathrm{~b})$ to $(4 \mathrm{~b})$ to make $\left(u_{x}\right)_{i}$ fourth-order
even with a non-uniform mesh.

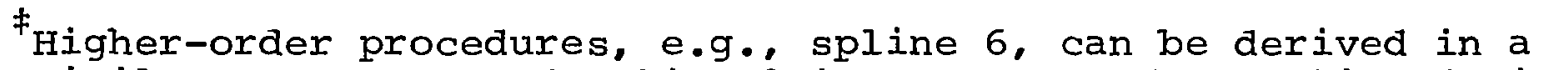
similar manner, and spline 2 is recovered from spline 4 with $\Delta$ set equal to zero.
} 
remains tridiagonal. Unlike spline 2, where the system can be reduced to that for $\mathrm{M}_{i}$ alone, the appearance of off-diagonal terms in (5b) restricts the reduction process to a $2 \times 2$ system in $\left(u_{i}, M_{i}\right)$.

For the linear Burgers equation the system is still of the form (3b) with

$$
\begin{aligned}
& \gamma_{1}=-\nu_{i}^{n+1} \theta \Delta t \sigma \Delta / 6 ; \quad \gamma_{a}=-\nu_{i}^{n+1} \theta \Delta t \Delta / 6 ; \\
& \alpha_{2}=-\nu_{i}^{n+1} \theta \Delta t(1-(1+\sigma) \Delta / 6) ; \\
& \rho_{2}=(1-\theta) \nu_{i}^{n+1} \Delta t(1-(1+\sigma) \Delta / 6) ; \\
& \delta_{1}=\nu_{i}^{n+1}(1-\theta) \Delta t \Delta / 6 .
\end{aligned}
$$

All other entries in $(3 c, 3 d)$ are unchanged.

IV. FINITE-DIFFERENCE THEORY/SPLINE 1

If the procedures given previously for spline 2 and spline 4 are repeated for a quadratic polynomial interpolation with both derivatives continuous (a quadratic spline of zero deficiency), we find on $\left[x_{i-1}, x_{i}\right]$,

$$
S_{\Delta}(x)=u_{i}\left(x-x_{i-1}\right) / h+u_{i-1}\left(x_{i}-x\right) / h+\left(u_{i}-u_{i-1}-h m_{i}\right)\left(x-x_{i-1}\right)\left(x_{i}-x\right) / h^{2},
$$

where

$$
s_{\Delta}\left(x_{i}\right)=u_{i}, s_{\Delta}\left(x_{i-1}\right)=u_{i-1}, s_{\Delta}^{\prime}\left(x_{i}\right)=m_{i},
$$

and

$$
M_{i}^{-}=s_{\Delta}^{\prime \prime}\left(x_{i}\right)=-2\left(u_{i}-u_{i-1}-m_{i} h\right) / h^{2}
$$


on $\left[x_{i}, x_{i+1}\right]$, with $s_{\Delta}\left(x_{i+1}\right)=u_{i+1}, s_{\Delta}\left(x_{i}\right)=u_{i}$ and $s_{\Delta}^{\prime}\left(x_{i}\right)=m_{i}$

we obtain

$$
M_{i}^{+}=S_{\Delta}^{\prime \prime}\left(x_{i}^{+}\right)=-2\left(u_{i}-u_{i+1}+m_{i} h\right) / h^{2}
$$

From the continuity of the second-derivative

$$
M_{i}^{+}=M_{i}^{-}
$$

and therefore

$$
m_{i}=\left(u_{i+1}-u_{i-1}\right) / 2 h
$$

The expression for $M_{i}$ becomes

$$
M_{i}^{+}=M_{i}^{-}=\left(u_{i+1}-2 u_{i}+u_{i-1}\right) / h^{2} \text {. }
$$

Therefore the quadratic spline of zero deficiency leads to the central difference expressions.

\section{STABILITY}

For the linear Burgers equation (3), with $\bar{u}, v$ constant, the interior point stability can be assessed with the von Neumann Fourier decomposition of the system (3) for $h_{i}=h=$ constant. With $v_{i+r}^{n}=v_{i}^{n} \operatorname{expI} w\left(x_{i}+r h\right), I=(-1)^{\frac{1}{2}}$, (3) becomes

$$
\mathrm{T}_{i} \gamma_{i}^{\mathrm{n}+1}=\mathrm{P}_{i} \gamma_{i}^{\mathrm{n}} \text { or } \gamma_{i}^{\mathrm{n}+1}=\mathrm{G}_{i} \gamma_{i}^{\mathrm{n}} \text {. }
$$

where $G_{i} \times T_{i}^{-1} P_{i}$ is the amplification matrix. The von Neumann condition necessary for the suppression of all error growth 
requires that the spectral radius $\rho\left(G_{i}\right) \leq I$. The eigenvalues of $G_{i}$ are $\lambda_{i}$.

For the one-dimensional equation (3), three numerical procedures were considered: (I) convection $\left(m_{i}\right)$ and diffusion $\left(M_{i}\right)$ explicit, (ii) convection explicit, diffusion implicit (two steps required for inviscid stability), and (iii) diffusion and convection implicit. With explicit convection, (i) or (ii), both divergence and nondivergence forms of the equations have been evaluated in Refs. 1 and 2 .

The stability conditions imposed on these schemes is determined from

$$
\left|\lambda_{i}\right| \leq 1
$$

(i) Explicit convection and diffusion: $\theta=0$ in $(2 a, 3)$.

Spline $2^{1,2}:\left|\lambda_{i}\right|^{2}=\left(1-6 \beta(1-\cos \varphi)(2+\cos \varphi)^{-1}\right){ }^{a}+c^{2} \Phi^{2} \leq 1$, where $\beta=\nu \Delta t / h^{2}, \quad c=\bar{u} \Delta t / h, \Phi=3 \sin \varphi /(2+\cos \varphi), \varphi=\omega h$. Necessary stability limits are

(a) $\beta \leq 1 / 6$,

(b) $\mathrm{C} \leq(3)^{-\frac{1}{2}}$,

(c) $R_{C}=c / \beta=\bar{u} h / \nu \leq 2(3)^{\frac{1}{2}}$.

These results are more restrictive than the limits found for the forward time central space explicit finite-difference method ${ }^{12}$ or spline 1, which are
(a) $B \leq 1 / 2$,
(b) $c \leq 1$,
(c) $R_{c} \leq 2$. 
Spline 4: $|\lambda|^{2}=(1-(5+\cos \varphi)(1-\cos \varphi) \beta /(2+\cos \varphi))^{2}+(3 \operatorname{csin} \varphi /$ $(2+\cos \varphi))^{2}<1$, so that necessary stability limits are

(a) $B \leq 1 / 4$, (b) $c \leq(10)^{\frac{1}{2}} / 6$, (c) $R_{C} \leq(40)^{\frac{1}{2}} / 3$.

Once again these conditions are somewhat more restrictive than those obtained with second-order finite-differences (7b). The Padé finite-difference limitation $c \leq(6)^{\frac{1}{2}} / 6$ is even more restrictive, see Appendix of Refs. 1 and 2. It is significant that in all cases the explicit method is unconditionally unstable for inviscid flow; i.e., $\beta=0$.

(ii) Two-step explicit integration (2b):

This procedure, which alleviates the inviscid instability found in (i), is a two-step predictor-corrector method (see Refs. I and 2) and is similar to the Brailovskaya ${ }^{11}$ two-step finite-difference technique. For $\beta=0$, we obtain

$$
c \leq \Phi_{\min }^{-1}=\left[(2+\cos \varphi)(3 \sin \varphi)^{-1}\right]_{\min }=(3)^{-\frac{1}{2}} \text {. }
$$

This result is more restrictive than the $c \leq l \mathrm{CFL}$ condition found for the Brailovskaya finite-difference method.

For $\beta \neq 0$, the effect of diffusion when treated implicitly is to improve the inviscid stability limitation. For $\overline{\mathrm{u}} \rightarrow 0$, the method is unconditionally stable ${ }^{1,2}$. Since the convection terms are unchanged, spline 4 has the same stability condition.

\section{(iii) Implicit convection and diffusion:}

The spline 2 and spline 4 procedures are unconditionally stable if $\theta \geq 1 / 2$ in Eqs. (2), (3). 


\section{(iv) SADI:}

In Ref. 1, the interior point stability analysis is extended to the two-dimensional SADI procedure; unconditional stability is demonstrated.

Although the implicit procedures lead to unconditionally stable formulations, as with finite-difference methods, the tridiagonal system may not be diagonally dominant. In this case the inversion algorithm ${ }^{3}$ may lead to large error growth. Diagonal dominance can be achieved by a spline adaptation of the finite-difference procedure given in Ref. 12. For all the problems treated here this modification is unnecessary. In other applications it will play a significant role if accurate solutions are to be obtained.

\section{RESULTS}

Several model problems have been considered in order to evaluate the cubic spline collocation methods presented herein. For each of these problems an analytic solution or reliable numerical solution is available for comparison purposes. Spline interpolation (spline 2 and spline 4) is used to approximate the spatial gradients. For the one-dimensional Burgers equation the integration procedure outlined in section II is adopted. Implicit or two-step explicit methods are used. For the two-dimensional diffusion equation, solutions are obtained with the SADI formulation. The Laplace equation in cartesian and polar coordinates is evaluated with a spline successive approximation procedure. Finally, the similarity equations for the flat plate boundary-layer 
and the two-dimensional stagnation point are solved by direct integration of the resulting two-point boundary value problems.

Solutions are obtained with both uniform and non-uniform meshes. Three-point finite-difference calculations are included in order to assess the relative increase in accuracy associated with the higher-order procedures. The results are presented in tabular form so that meaningful comparisons are possible.

A. Burgers Equation

The nonlinear Burgers equation (3a), with $x=\bar{x}, \vec{u}=u(\bar{x}, t)$ and $x=\bar{x}-(1 / 2) t$, becomes

$$
u_{t}+(u-1 / 2) u_{x}=u_{x x}
$$

with $\nu$ constant and the boundary conditions

$$
u \rightarrow 1 \text { as } x \rightarrow-\infty \text { and } u \rightarrow 0 \text { as } x \rightarrow \infty
$$

The steady state solution of (8a) is

$$
u=[I-\tanh (x / 4 v)] / 2
$$

Spline 2 and the finite-difference solutions of (8) have been discussed in Refs. 1 and 2 . Both implicit ${ }^{*}$ and two-step explicit integration techniques, as outlined in section II, have been applied successfully ${ }^{1,2}$. Spline 4 solutions have now been obtained with the implicit* and/or two-step procedures of (2a). (2b). The system (3) with the coefficients (6) are considered. In the actual calculations the $3 \times 3$ system (3) is

*The nonlinear coefficient u is treated iteratively or with quasi-linearization 1,2 . 
reduced to a $2 \times 2$ system. $m_{i}$ in $v_{i}$ is eliminated with (le) or (1f). The boundary conditions (8b) on $u_{1}, u_{N+1}$ are specified at $\mathrm{x}=\mathrm{x}_{\max }$, with $\mathrm{x}_{\max } \geq 3$. The boundary conditions on $\mathrm{M}_{i}$ are obtained from the third-order accurate relation

$$
\left(u_{x x}\right)_{i+1}-\left(u_{x x}\right)_{i}=M_{i+1}-M_{i}
$$

where $i=1$ or $\mathrm{N}$.

The boundary condition (9a) can be applied in two forms. These are outlined for the boundary $i=1$ :

(a) $\quad\left(u_{x x}\right)_{1}=\left(u_{x x}\right)_{2}-\left(M_{2}-M_{1}\right)$

With $\left(u_{x x}\right)_{2}$ evaluated from $(5 c)$, we obtain

$$
\left(u_{x x}\right)_{I}=M_{1}+(\Delta / 6)\left(M_{3}-(I+\sigma) M_{2}+\sigma M_{1}\right),
$$

where $\sigma=h_{3} / h_{2}$. From the governing equation (8a),

$$
\left(u_{x x}\right)_{1}=\left(u_{x}\right)_{1} / 2 v=m_{1} / 2 \nu
$$

so that with (If),

$$
\mathrm{m}_{1}=-\mathrm{h}_{2} \mathrm{M}_{1} / 3-\mathrm{h}_{2} \mathrm{M}_{2} / 6+\left(\mathrm{u}_{2}-1\right) / \mathrm{h}_{2}
$$

and (9b) becomes

$$
M_{1}\left(2 \nu+\sigma \nu \Delta / 3+h_{2} / 3\right)+M_{2}\left(h_{2} / 6-\nu(1+\sigma) \Delta / 3\right)+(\nu \Delta / 3) M_{3}-u_{2} / h_{2}=-1 / h_{2} \cdot
$$

(b) An alternate form of (9a), relating only the two points, $i=1$ and $i=2$, can be derived by evaluating $\left(u_{x x}\right)_{a}$ from (8a). The temporal discretization is given by (2a). We obtain

$$
a_{1} M_{1}^{n+1}+a_{2} M_{2}^{n+1}+a_{3} u_{2}^{n+1}=a_{4}
$$


where

$$
\begin{aligned}
& a_{1}=\Delta t\left(v+\left(u_{2}^{n}+0.5\right) h_{2} / 6\right) ; \\
& a_{2}=-\Delta t\left(v-\left(u_{2}^{n}-0.25\right) h_{2} / 3\right) ; \\
& a_{3}=\left(1+\Delta t\left(u_{2}^{n}-1\right) / h_{2}\right) ; \\
& a_{4}=\left(u_{2}^{n}+\left(u_{2}^{n}-1\right) \Delta t / h_{2}\right)
\end{aligned}
$$

For spline $4, \Delta=\left(1+\sigma^{3}\right) / \sigma(1+\sigma)^{2}$. For spline 2, set $\Delta=0$ so that (9c) is second-order accurate. Eq. (9b) is third-order accurate for both spline 2 and spline 4. Similar relations are obtained for the other boundary, where $u_{N+1}=0$.

The condition (9c) is independent of the time step $\Delta t$ and somewhat less cumbersome. It was found that the accuracy of the solutions and the time to attain a converged steady state solution were virtually insensitive to the choice of the boundary condition (9c) or (9d). This conclusion remains unchanged if the higher order effects in (9b), i.e.. those terms multiplied by $\Delta$, are treated explicitly in (9c). In this way (9c) reduces to a two-point implicit formula. In several cases the simpler spline 2 boundary conditions were applied with the spline 4 procedure; the solutions always fell between the sesults of spline 2 and spline 4, but generally closer to those of spline 4. Therefore, if simplicity of boundary conditions is desired this is a reasonable approximation.

Typical results are shown, for $\nu=1 / 8,1 / 16,1 / 24$ on Tables 1-5. The increase in accuracy as one progresses from the finitedifference results to those of spline 2 and finally to spline 4 
is apparent. This is particularly true with the non-uniform meshes of Tables 2 and 3 . For the conditions of Table 3 , the finite-difference calculations with the two-step explicit procedure did not converge. An oscillatory behavior was observed after 3200 iterations. In certain cases, where $h_{i}$ is relatively large, the nature of the truncation errors $(4 a, 4 b)$ of spline 2 and spline 4 is such that a local value obtained with spline 2 may be as accurate or more accurate than that obtained with spline 4. These are exceptional cases, however, and never occur for $h_{i}<l$. A percentage error plot for the results of Table 1 is shown on Figure $1 . u_{e}(x)$ denotes the exact solution (8c). Solutions for other $v$ values are of a similar nature and therefore have not been included here.

B. Linear Burgers Equation

Consider the equation

$$
u_{x}+\nu u_{x x}=0, \text { on } 0 \leq x \leq 1
$$

with boundary conditions $u(1)=1$ and on $x=0, v u_{x}+u=0$. The exact solution is $u_{e}(x)=\exp (1-x) / v$. In some unpublished work by George J. Fix*, it was shown that with this flux boundary condition linear finite element theory naturally satisfies the required conservation condition at the boundary and therefore leads to more accurate solutions than obtained with non-divergence versions of spline 2 or conventional finite-difference theory. If finite-difference theory is developed in divergence or conservation form, the resulting equations are identical with

\footnotetext{
*Institute for Computer Applications in Science and Engineering
} 
those of the Iinear, second-order accurate, finite element method. If spline 2 is recast in divergence form the solutions are considerably more accurate than the non-conservation results and also improve upon the conservation finite element (finitedifference) calculations. Therefore, with the flux boundary condition it appears that divergence form may be required if accurate spline solutions are to be obtained. On the other hand, if a modified derivative boundary condition was considered in lieu of the flux condition, the sensitivity to divergence form was no longer apparent. It is possible therefore that the flux condition represents a singular case.

The governing systems of equations and the boundary conditions for the different formulations are as follows: Finite-Difference/Non-Divergence Form

$$
v\left(u_{i+1}+u_{i-1}-2 u_{i}\right) / h+\left(u_{i+1}-u_{i-1}\right) / 2=0
$$

At $x=1$,

$$
\mathrm{u}_{\mathrm{N}}=I
$$

At $x=0, \quad v\left(u_{1}-u_{-1}\right) / 2 h+u_{0}=0$

Eliminating $u_{-1}$ from (10c) with the difference equation (10a), we obtain

$$
2 v^{2}\left(u_{1}-u_{0}\right) / h^{2}+u_{0}(2 v / h-1)=0
$$

Finite-Difference/Divergence Form E Einite Element

$$
\left(v u_{x}+u\right)_{x}=0
$$

Therefore, $\quad\left(v u_{x}+u\right)_{i+\frac{1}{2}}=\left(v u_{x}+u\right)_{i-\frac{1}{2}}$ 
or

$$
v\left(u_{i+1}+u_{i-1}-2 u_{i}\right) / h+\left(u_{i+1}-u_{i-I}\right) / 2=0
$$

The governing equation (1la) is identical with the non-divergence equation (10a). The alterations appear in the boundary conditions

At $x=1, \quad u_{N}=1$

At $x=0, \quad\left(v u_{x}+u\right)_{\frac{1}{2}}=0$. Therefore,

$$
v\left(u_{1}-u_{0}\right) / h+\left(u_{1}+u_{0}\right) / 2=0
$$

The boundary condition (IIb) differs from the non-divergence condition (10d).

Spline 2/Non-Divergence Form

The governing equation (12a) is combined

$$
v M_{i}+m_{i}=0
$$

with the spline relations (1). The boundary conditions are

$$
u_{N}=1, \quad v m_{0}+u_{0}=0
$$

\section{Spline 2/Divergence Form}

The governing equation (13a) is combined

$$
(v m+u)_{i+1}=(v m+u)_{i-1}
$$

with the spline relation (IC). The boundary conditions are

$$
u_{N}=1
$$

and

$$
v m_{0}+u_{0}+\alpha\left(v m_{1}+u_{1}\right)=0
$$

where $\alpha=0$ corresponds to the exact boundary value and $\alpha=1$ corresponds to an averaged boundary condition. 
The results of these calculations are shown on Table 6 . It is seen that the non-conservation (NC) solutions with ten mesh points $(\mathrm{N}=10)$ are rather poor when compared with either the finite element or spline 2 conservation (c) solutions. It is significant that the spline divergence solutions, for both $\alpha=0$ and $\alpha=1$, are considerable improvements over the finiteelement results. As the number of mesh points increases the non-divergence solutions do show some improvement, with spline 2 more accurate than finite-differences, but these results are still less accurate than finite-element solutions. The ten point spline 2 divergence form $\alpha=1$ solutions are about as accurate as the 50 point finite element results.

Also shown on the table are ten point solutions with somewhat modified derivative conditions at $x=0$. The exact solution is unchanged. These derivative boundary conditions were treated in much the same manner as the flux condition for each of the procedures. For the finite-element solutions an average condition was applied. Significantly the large differences between divergence solutions no longer occur. The spline solutions are always the most accurate, with a small increase in accuracy when divergence form is assumed.

c. Linear Corner Flow

The two-dimensional diffusion equation

$$
u_{t}=\frac{1}{R_{e}}\left(u_{x x}+u_{y y}\right), u=u(t, x, y)
$$

with the initial condition $u(0, x, y)=0$ and boundary conditions 


$$
\begin{aligned}
& u(t>0,0, y \geq 0)=1, u(t>0, x \geq 0,0)=1 \\
& u(t, x, y) \rightarrow 0 \text { as } x, y \rightarrow \infty
\end{aligned}
$$

has the exact solution

$$
u=1-\operatorname{erf} X \operatorname{erf} Y \text {, }
$$

where $X=\frac{X}{2}\left(R_{e} / t\right)^{\frac{1}{2}}, Y=\frac{Y}{2}\left(R_{e} / t\right)^{\frac{1}{2}}$.

This solution describes the impulsive motion of a rightangled corner formed by two infinite flat plates and results of the SADI spline 2 calculation have been presented in Refs. 1,2. The SADI procedure for the diffusion equation (14a) for both spline 2 and spline 4 is given as follows:

Step l: $u_{i j}^{n+\frac{1}{2}}=u_{i j}^{n}+\left(\left(u_{x x}\right)_{i j}^{n+\frac{1}{2}}+\left(u_{y y}\right)_{i j}^{n}\right) \Delta t /\left(2 R_{e}\right)$

step 2: $u_{i j}^{n+1}=u_{i j}^{n+\frac{1}{2}}+\left(\left(u_{x x}\right)_{i j}^{n+\frac{1}{2}}+\left(u_{y y}\right)_{i j}^{n+1}\right) \Delta t /\left(2 R_{e}\right)$

where

$$
\left(u_{x x}\right)_{i j}=M_{i j}+\left(\Delta_{x} / 6\right)\left(M_{i+1, j}-\left(1+\sigma_{x}\right) M_{i j}+\sigma_{x} M_{i-1, j}\right)
$$

and

$$
\left(u_{y Y}\right)_{i j}=L_{i j}+\left(\Delta_{y} / 6\right)\left(L_{i, j+I}-\left(I+\sigma_{y}\right) L_{i j}+\sigma_{y} L_{i, j-1}\right)
$$

$L_{i j}$ and $M_{i j}$ each satisfy a tridiagonal equation of the form (1b) .

$$
\begin{aligned}
& \Delta_{x}=\left(1+\sigma_{x}^{3}\right) / \sigma_{x}\left(1+\sigma_{x}\right)^{2} ; \Delta_{y}=\left(1+\sigma_{y}^{3}\right) / \sigma_{y}\left(1+\sigma_{y}\right)^{2} \\
& \sigma_{x}=h_{i+1} / h_{i} ; \sigma_{y}=k_{j+1} / k_{j} ; h_{i}=x_{i}-x_{i-1} ; k_{j}=y_{j}-y_{j-1} \cdot
\end{aligned}
$$

The spline 2 formulation is recovered with $\Delta_{x}=\Delta_{y}=0$. The boundary conditions for $u_{i}$ are given by (14b). The boundary conditions 
for $L_{i j}, M_{i j}$ are obtained from (16) with $\left(u_{x x}\right)_{i j}=\left(u_{y y}\right)_{i j}=0$ on the boundaries, or from (9a) with $\left(u_{x x}\right) i+1, j$ obtained from (14a).* The solution for step 1 is obtained with the tridiagonal $2 \times 2$ system for $M_{i j}$ and $u_{i j}$ as described by (15a) and (1b). A similar procedure for $\mathrm{L}_{i j}, \mathrm{u}_{i j}$ is required for step 2 . The solution for $R_{e}=1000$ is given on Table 7. A non-uniform $21 \times 21$ mesh with $\sigma_{x}=\sigma_{y}=1.5$ was prescribed. The step size $\Delta t=0.01$. The solution is shown for $t=2.0$. All of the solutions are reasonably good for this case, but once again the spline solutions are somewhat better.

D. Laplace Equation

The Laplace equation

$$
u_{x x}+u_{y y}=0 ; u=u(x, y)
$$

with the boundary conditions $u(0, y)=u(1, y)=0 ; u(x, 0)=\sin \pi x$; $\lim y \rightarrow \infty \quad u(x, y)=0$

has the solution

$$
u(x, y)=(\sin \pi x) \exp (-\pi y)
$$

This boundary layer-like problem was chosen in order to evaluate the accuracy of the spline procedures, in particular with non-uniform meshes, when large gradients exist only over a limited region. In addition, this problem will serve as a prototype for spline integration using successive approximation (relaxation) procedures.

\footnotetext{
* This procedure has been demonstrated for the Burgers equation by the discussion leading to (9d).
} 
Using the general expression for second derivatives (16), Eq. (17a) can be put into a spline form. With the tridiagonal relationship for $I_{i j}$ and $M_{i j}(I b)$, this leads to a $3 \times 3$ system for the vector $v_{i j}$ at all interior mesh points:

$$
\mathrm{A}_{i j} \mathrm{~V}_{i, j-1}+\mathrm{B}_{i j} \mathrm{~V}_{i j}+\mathrm{C}_{i j} \mathrm{~V}_{i, j+1}+\mathrm{D}_{i j} \mathrm{~V}_{i-1, j}+\mathrm{E}_{i j} \mathrm{~V}_{i+1, j}=0
$$

where

$$
\begin{aligned}
& V_{i j}=\left[\begin{array}{l}
u_{i j} \\
L_{i j} \\
M_{i j}
\end{array}\right] \\
& A_{i j}=\left[\begin{array}{ccc}
0 & \sigma_{y} \Delta_{y} / 6 & 0 \\
-6 / k_{j}^{2} & 1 & 0 \\
0 & 0 & 0
\end{array}\right] \text {, } \\
& B_{i j}=\left[\begin{array}{ccc}
0 & \left(1-\frac{1+\sigma_{y}}{6} \Delta_{y}\right) & \left(1-\frac{1+\sigma_{x}}{6} \Delta_{x}\right) \\
\frac{6}{k_{j}^{2}} \frac{\left(1+\sigma_{y}\right)}{\sigma_{y}} & 2\left(I+\sigma_{y}\right) & 0 \\
\frac{6}{h_{i}^{2}} \frac{1+\sigma_{x}}{\sigma_{x}} & 0 & 2\left(1+\sigma_{x}\right)
\end{array}\right] \\
& C_{i j}=\left[\begin{array}{ccc} 
& \Delta_{y} / 6 & 0 \\
\frac{-6}{\sigma_{y} k_{j}^{2}} & \sigma_{y} & 0 \\
0 & 0 & 0
\end{array}\right] ; D_{i j}=\left[\begin{array}{ccc}
0 & 0 & \frac{\sigma_{x} \Delta_{x}}{6} \\
0 & 0 & 0 \\
\frac{-6}{h_{i}^{2}} & 0 & 1
\end{array}\right]
\end{aligned}
$$




$$
E_{i j}=\left[\begin{array}{ccc}
0 & 0 & \Delta_{x} / 6 \\
0 & 0 & 0 \\
\frac{-6}{\sigma_{x} h_{i}^{2}} & 0 & 1
\end{array}\right]
$$

The solution is obtained with a successive point relaxation procedure,

$$
v_{i j}^{(k+I)}=B_{i j}^{-I}\left[A_{i j} v_{i, j-I}^{(k+1)}+C_{i j} v_{i, j+1}^{(k)}+D_{i j} v_{i-1, j}^{(k+I)}+E_{i j} v_{i+1, j}^{(k)}\right]
$$

where the superscript $k$ represents the iteration parameter. The system is diagonally dominant and the eigenvalues of the amplification matrix, see section $V$, are all less than or equal to one. The results of this compuation are presented in Table 8. The values of $u_{y}(.5,0)$ and $u\left(.5, h_{2}\right)$ are compared with the exact solution (17b). Also included in this table are the results obtained with the three-point finite-difference approximation for $u_{x x}$ and $u_{Y y}$. In order to make a more definitive comparison between the spline and finite-difference solutions, the surface value of $u_{y}$ in the latter case was obtained by spline fitting the numerical values of $u(x, y)$. In one case noted on Table 8 , a three-point end difference formula was applied. All of the calculations were performed with 10 mesh points in the normal or $y$-direction. In certain cases, spline 2 was used in the $y-$ direction and spline 4 in the $\mathrm{x}$-direction. These solutions are noted accordingly.

The spline 4 results are the most accurate in all cases. For a uniform mesh the finite-difference and spline 2 results 
are of equal accuracy as there are no convection effects in the problem. Moreover, if the spline 2 and the finite-difference solutions are averaged, the spline 4 results are closely approximated. For a non-uniform mesh the improved accuracy of spline 2 over the finite-difference approximation is now apparent. The spline 4 results are remarkably accurate with $\sigma=1.7$, $h_{a}=0.1$ and $y_{\max }=28.66$. For this mesh there are only four points in the region $0 \leq y \leq l$ as compared with a uniform mesh $(h=0.1)$ and ten points. The coarse mesh, spline 4 results are more accurate than the uniform mesh finite-difference solutions.

The $1.7 / .2$ notation for $\sigma$ means that $\sigma=1.7$ for $h_{i}<0.2$. For $h_{i} \geq 0.2$, $\sigma$ becomes unity. In this way the mesh width does not exceed a specified maximum value. This type of mesh alignment is useful in boundary layer problems, where a fine grid is desired near the surface, and a uniform but coarser mesh is required in the outer inviscid regions. This procedure is also applied for the boundary layer solutions in section VI.F. An error plot is given on Figure 2 .

E. Potential Flow over a Circular cylinder

The governing equation in cylindrical coordinates for the potential flow over a circular cylinder is given by

$$
u_{r r}+\frac{1}{r} u_{r}+\frac{1}{r^{2}} u_{\theta \theta}=0
$$

The boundary conditions are $u_{r}(1, \theta)=0$ and $\underset{r \rightarrow \infty}{\lim } u(r, \theta) \rightarrow r \cos \theta$. The exact solution of Eq. (20) with these boundary conditions is $\mathrm{u}=\left(\mathrm{r}+\frac{I}{\mathrm{r}}\right) \cos \theta$. Eqs. (17a) and (20) differ only by the appearance 
of the $u_{r}$ term which is discretized by the relations (le) or (1f). The resulting $3 \times 3$ system for $u_{i j}, I_{i j}$ and $M_{i j}$ is of the form (19). The coefficient matrices in the present case will be somewhat altered by the $u_{r}$ term.

The results of the iterative solution are presented in Tables 9-11. As in the previous examples, the finite-difference solutions are obtained by using three-point central difference formulas. In Table 11, the slip velocity on the fore surface of the cylinder is presented. The superiority of the spline solutions over those resulting from finite-difference discretization is evident. It should be noted that the slip velocity in the finite-difference case is obtained by using a three-point central difference formula, while the spline solutions require only the two-point formula (le). The higher accuracy of the two-point spline formula over the three-point finite-difference relations can be of considerable importance for problems with derivative boundary conditions.

F. Similarity Boundary Layers

The boundary layer equations for the flow over a flat plate $(\beta=0)$ and the two-dimensional stagnation point $(\beta=1)$ can be reduced to the following ordinary differential system by using appropriate similarity transformations! ${ }^{14}$.

$$
\begin{aligned}
& u^{\prime \prime}+f u^{\prime}+\beta\left(1-u^{2}\right)=0 \\
& f^{\prime}=u
\end{aligned}
$$

The boundary conditions are

$$
f(0)=0, u(0)=0, \lim _{x \rightarrow \infty} u(x)=1.0
$$


Accurate numerical solutions have been reported in the literature [see Rosenhead ${ }^{14}$ ].

In the spline 2 and spline 4 formulation, Eq. (2la) is reduced to a $2 \times 2$ system for $u_{i}$ and $M_{i}$ and the two-point boundary value problem is solved subject to (2lc). For the first-order equation (21b), we obtain the following spline approximation from (If) :

$$
f_{i+1}=f_{i}+h_{i+1} u_{i}+\frac{h_{i+1}^{2}}{3}\left(M F_{i}+.5 M F_{i+1}\right)
$$

where $\mathrm{MF}_{i}=\left(u^{\prime}\right)_{i}$ for spline 2. For spline 4, the following relation to evaluate $\mathrm{MF}_{i}$ is easily derived from (le) and (If):

$$
\begin{aligned}
& M F_{i+1}+M F_{i}=2\left((u)_{i+1}-(u)_{i}\right) / h_{i+1} \\
& M F_{O}=\left(u^{\prime}\right)_{0}
\end{aligned}
$$

Eqs. (22) and (23) give rise to an initial value problem for $f_{i}$ and $\mathrm{MF}_{i}$ which is solved by a marching procedure. Eq. (22) leads to third-order accurate expression for $f_{i}$; therefore, for non-uniform meshes and third-order accurate solutions, this approximation is adequate even for spline 4. For the finitedifference solutions, a second-order accurate two-point formula for $f_{i}$, which is consistent with the accuracy of the overall scheme, is obtained with the trapezoidal rule. For $\beta=1$, the nonlinear term $u^{2}$ is treated by quasilinearization so that

$$
\left(u^{k+1}\right)^{z}=u^{(k)}\left(2 u^{(k+1)}-u^{(k)}\right) \text {. }
$$

$\mathrm{k}$ is the iteration parameter. 
The results of these computations for both uniform as well as non-uniform meshes are tabulated in Tables 12-16. The shear at the wall is proportional to $f^{\prime \prime}(0)$ and this term has been evaluated for a variety of meshes. The results are given on Tables 15 and 16 for $\beta=0$ and $\beta=1$, respectively. Finitedifference solutions for $u_{i}$ are obtained by using the threepoint central difference approximation.

As noted previously, the notation $\sigma=1.8 / 1$ means that $\sigma=1.8$ until $h_{i}$ reaches 1.0 , at which point $\sigma=1.0 . h_{2}$ is the first mesh width off the wall $x=0 ; N$ is the total number of mesh points. $N_{6}$ is the number of mesh points in the boundary layer defined by $x \leq 6$. At $x=6,|u-1.0|<10^{-5}$.

\section{$\beta=0$ Blasius solution:}

The spline 4 solution for $N=61, h_{2}=0.1$ and $\sigma=1.0$ is almost identical with the "exact" solution of $f^{\prime \prime}(0)=0.469600 . .^{14}$ If spline 2 boundary conditions are used with a spline 4 interior point formulation, $f^{\prime \prime}(0)=0.469608$. As previously noted, this value lies between the spline 2 and spline 4 results. With $\sigma=1.8 / 2$, $h_{z}=0.5, N=21$ and only 5 points in the boundary layer $\left(N_{6}=5\right)$, the spline 2 value of $f^{\prime \prime}(0)$ is in error by only $2 \%$. For the larger $h_{z}$ values the spline 2 solutions are ever more accurate than those found with spline 4. Similar behavior was observed with Burgers equation in section VI.A. An error plot is given on Figure 3 .

\section{$\underline{\beta}=1$ stagnation point flow:}

For $\beta=0$, the exact solution has $u^{\prime \prime \prime}(0)=u^{i v}(0)=0$ and therefore the inherent lower-order accuracy of the finite-difference 
calculation is somewhat obscured near the wall $x=0$. For the stagnation point solution where $f^{\prime \prime}(0)=1.232588$, the improvement associated with the spline formulation is clearly demonstrated. Therefore, it would appear that spline integration should be extremely useful for boundary layer problems.

G. Non-Similar Boundary Layer Analysis

As a final test of the spline procedures the constant pressure boundary layer equations written in physical variables $(x, y)$ were considered:

$$
\begin{aligned}
& u_{x}+v u_{y}=R_{e}^{-1} u_{y y} \\
& u_{x}+v_{y}=0
\end{aligned}
$$

The boundary conditions are

$$
\begin{array}{ll}
\mathrm{y}=0: & \mathrm{u}=\mathrm{v}=0 \\
\mathrm{y}>1 & \mathrm{u} \rightarrow \mathrm{I}
\end{array}
$$

The initial conditions were given by

$$
\begin{aligned}
& u(0, y)=1.0, \quad y \neq 0 \text { and } u(0,0)=0 \\
& v(0, y)=0
\end{aligned}
$$

The equations were integrated for a Reynolds number $R e=10^{5}$ and a non-uniform mesh of ten points normal to the surface. The solution for the normalized skin friction is shown on Figure 4. The value $\mathrm{N}_{6}$ denotes the actual number of points within the boundary layer. The same criteria of section VI.F was applied. As the boundary layer grows with distance $x, N_{6}$ increases. With 6 to 7 points in the final boundary layer profiles, the 
spline 4 solutions are quite accurate.

\section{SUMMARY}

It has been demonstrated that higher-order calculation procedures using cubic spline collocation provide accurate solutions to a number of model problems. The spline methods termed spline 2 and spline 4 can be used for two-point boundary value problems, as well as implicit, explicit, two-step, ADI and iterative integration procedures.

spline 4 is fourth-order accurate with a uniform mesh and third-order with a moderate non-uniform mesh. Spline 2 is second-order accurate for diffusion terms and fourth-order (third-order) for convection with a uniform (non-uniform) mesh. Derivative boundary values are obtained directly without the need for end differencing. For implicit linear systems, the spline methods remain unconditionally stable.

The results confirm the higher-order accuracy of the spline methods and lead to the hopeful conclusion that accurate solutions for more practical flow problems can be obtained with relatively coarse non-uniform meshes.

There has been no attempt to optimize the temporal integration procedure so as to minimize computer times or increase temporal accuracy. The finite-difference calculations run $20 \%$ to $25 \%$ faster than the spline integrations. When spline fitting is used to evaluate finite-difference derivatives, as in 
section VI.C, the computer times are comparable. It is anticipated that the reduced mesh requirements with these spline methods will result in a net improvement in computer storage and time.

\section{REFERENCES}

1. Rubin, S.G. and Graves, R.A.., "Cubic Spline Approximation for Problems in Fluid Mechanics". NASA TR R-436, 1975.

2. Rubin, S.G. and Graves, R.A., "Viscous Flow solutions with a Cubic Spline Approximation". Computers and Fluids, $\underline{3}$, 1. pp. 1-36, 1975 .

3. Ahlberg, J., Nilson, E. and Walsh, J., The Theory of Splines and Their Applications. Academic Press, New York, 1967.

4. Papamichael, N. and Whiteman, J., "A Cubic Spline Technique for the one-Dimensional Heat Conduction Equation". J. Inst. Math. Appl.. 11, pp. 111-113, 1973.

5. Peters, N.. "Boundary-Layer Calculations by a Hermitian Finite Difference Method". Fourth Int'1. Conference on Numerical Methods in Fluid Mechanics, Boulder, Colorado, 1974.

6. Collatz, L., The Numerical Treatment of Differential Equations. Springer-Verlag, Berlin, 3rd edition, 1960.

7. Orszag, S. and Israeli, M., Numerical Simulation of Viscous Incompressible Flows. Annual Review of Fluid Mechanics, 6 , 1974.

8. Hirsh, R., "Higher-Order Accurate Difference Solutions of Fluid Mechanics Problems by a Compact Differencing Technique". J. Comp. Phys, 19, 1, Sept. 1975. 
9. Krauss, E., Hirshel, E. H. and Kordulla, W., "Fourth Order "Mehrstellen"-Integration for Three-Dimensional Turbulent Boundary Layers". Proc. of AIAA Computational Fluid Dynamics Conference, Palm Springs, Calif., pp. 92-102, 1973.

10. Fyfe, D. J., "The Use of Cubic Splines in the Solution of Two-Point Boundary Value Problems". Comput. J., 12, pp. 188-192, 1969 .

11. Brailovskaya, I., "A Difference Scheme for Numerical Solution of the Two-Dimensional Nonstationary Navier-Stokes Equations for a Compressible Gas". Soviet Physics-Doklady, 10, pp. 107-110, 1965.

12. Khosla, P. K. and Rubin, S. G., "A Diagonally Dominant SecondOrder Accurate Implicit Scheme". Computers and Fluids, $\underline{2}$, 2, pp. 207-209, 1974 .

13. Roache, P., Computational Fluid Dynamics. Hermosa Publishers, Albuquerque, N.M., 1972 .

14. Rosenhead, L., Laminax Boundary Layers, Oxford at the Clarendon Press, England, 1963. 
TABLE ।

SOLUTION OF BURGERS EQUATION $\nu=1 / 8, \sigma=1.0,31$ EQUALLY

SPACED POINTS

\begin{tabular}{|c|c|c|c|c|}
\hline 1 & F. D. & SPLINE 2 & SPLINE 4 & EXACT \\
\hline 0 & 0.5000 & 0.5000 & 0.5000 & 0.5000 \\
\hline-0.2 & 0.6999 & 0.6860 & 0.6900 & 0.6900 \\
\hline-0.4 & 0.8447 & 0.8290 & 0.8322 & 0.8320 \\
\hline-0.6 & 0.9269 & 0.9160 & 0.9170 & 0.9170 \\
\hline-0.8 & 0.9673 & 0.9620 & 0.9609 & 0.9610 \\
\hline-1.0 & 0.9857 & 0.9830 & 0.9820 & 0.9820 \\
\hline-1.2 & 0.9938 & 0.9930 & 0.9918 & 0.9920 \\
\hline-1.4 & 0.9973 & 0.9970 & 0.9963 & 0.9960 \\
\hline-1.6 & 0.9988 & 0.9990 & 0.9983 & 0.9980 \\
\hline-1.8 & 0.9995 & 0.9990 & 0.9993 & 0.9990 \\
\hline
\end{tabular}




\section{TABLE 2}

SOLUTION OF BURGERS EQUATION $\nu=1 / 8, \sigma=1.2,15$ POINTS

\begin{tabular}{|c|c|c|c|c|}
\hline$X$ & F. D. & SPLINE 2 & SPLINE 4 & EXACT \\
0 & 0.5000 & 0.5000 & 0.5000 & 0.5000 \\
-0.3859 & 0.9510 & 0.8214 & 0.8297 & 0.8240 \\
-0.8494 & 1.0030 & 0.9778 & 0.9654 & 0.9676 \\
-1.4060 & 0.9990 & 1.0004 & 0.9951 & 0.9964 \\
-2.0750 & 1.0 & 1.0 & 0.9989 & 0.9997 \\
-2.8770 & 1.0 & 1.0 & 0.9999 & 1.0 \\
-3.8420 & 1.0 & 1.0 & 0.9996 & 1.0 \\
-5.000 & 1.0 & 1.0 & 1.0 & 1.0 \\
\hline
\end{tabular}




\section{TABLE 3}

SOLUTION OF BURGERS

EQUATION: $\nu=1 / 8, \sigma=1.8$,

15 POINTS

\begin{tabular}{|c|l|l|l|}
\hline$X$ & SPLINE 2 & SPLINE 4 & EXACT \\
\hline 0 & 0.5000 & 0.5000 & 0.5000 \\
-0.0662 & 0.5740 & 0.5691 & 0.5659 \\
-0.1855 & 0.6986 & 0.6858 & 0.6774 \\
-0.4001 & 0.8695 & 0.8452 & 0.8321 \\
-0.7864 & 1.0012 & 0.9689 & 0.9587 \\
-1.4818 & 1.0165 & 1.0083 & 0.9973 \\
-2.7334 & 1.0267 & 1.0257 & 1.0 \\
-4.9864 & 1.0 & 1.0 & 1.0 \\
\hline
\end{tabular}




\section{TABLE 4 SOLUTION OF BURGERS EQUATION: $\nu=1 / 16, \sigma=1.0$, 19 EQUALLY SPACED POINTS}

\begin{tabular}{|c|c|c|l|l|}
\hline$X$ & F. D. & SPLINE 2 & SPLINE 4 & EXACT \\
\hline 0 & 0.5000 & 0.5000 & 0.5000 & 0.5000 \\
-0.2 & 0.9000 & 0.8231 & 0.8356 & 0.8320 \\
-0.4 & 0.9878 & 0.9641 & 0.9617 & 0.9608 \\
-0.6 & 0.9986 & 0.9952 & 0.9916 & 0.9918 \\
-0.8 & 0.9998 & 0.9995 & 0.9982 & 0.9983 \\
-1.0 & 1.0 & 0.9999 & 0.9996 & 0.9997 \\
-1.2 & 1.0 & 1.0 & 0.9999 & 0.9999 \\
\hline
\end{tabular}




\section{TABLE 5 SOLUTION OF BURGERS EQUATION: $\nu=1 / 24, \sigma=1.2$, 31 POINTS}

\begin{tabular}{|c|c|c|c|}
\hline$x u$ & SPLINE 2 & SPLINE 4 & EXACT \\
\hline 0 & 0.5000 & 0.5000 & 0.5000 \\
\hline-0.0688 & 0.6936 & 0.6957 & 0.6955 \\
\hline-0.1514 & 0.8618 & 0.8606 & 0.8602 \\
\hline-0.2505 & 0.9586 & 0.9526 & 0.9529 \\
\hline-0.3695 & 0.9928 & 0.9876 & 0.9883 \\
\hline-0.5122 & 0.9995 & 0.9975 & 0.9979 \\
\hline-0.6835 & 1.0 & 0.9996 & 0.9997 \\
\hline-0.8890 & 1.0 & 1.0 & 1.0 \\
\hline-1.1356 & 1.0 & 1.0 & 1.0 \\
\hline-4.9582 & 1.0 & 1.0 & 1.0 \\
\hline
\end{tabular}


TABLE 6 LINEAR BURGERS EQUATION

\begin{tabular}{|c|c|c|c|}
\hline $\mathrm{N}$ & FINITE-ELEMENT & SPLINE & FINITE - DIFFERENCE \\
\hline 10 & 165.38 & $37.378(N C)$ & 14.7 \\
\hline 25 & 150.923 & 100.632 (NC) & \\
\hline 50 & 149.034 & $132.668(\mathrm{NC})$ & \\
\hline 100 & 148.568 & 144.137 (NC) & 136.022 \\
\hline 10 & - & 138.6 (C) & - \\
\hline 10 & - & 149.115 (C AVG.) & - \\
\hline
\end{tabular}

$\left(\nu u_{x}+2 u\right)=e^{1 / \nu}$ AT THE BOUNDARY

10 165.38

151.552 (NC)

148.486 (C)

$\left(\nu u_{x}\right)=-e^{1 / \nu}$ AT THE BOUNDARY

10 165.38 145.442 (NC) 139.296 148.342 (C) 
TABLE 7 LINEARIZED CORNER FLOW

\begin{tabular}{|c|c|c|c|c|c|c|c|}
\hline METHOD & $x$ & 0.0025 & 0.0081 & 0.0493 & 0.2575 & 1.3117 & 6.6485 \\
\hline $\begin{array}{l}\text { EXACT SOLUTION } \\
\sigma=1.5, \Delta t=0.01 \\
k_{2}=h_{2}=.001 \\
N_{x}=N_{y}=21 \\
t=2.0\end{array}$ & $\begin{array}{l}0.0025 \\
0.0081 \\
0.0493 \\
0.2575 \\
1.3117 \\
6.6485 \\
\end{array}$ & \begin{tabular}{|l|}
0.9990 \\
0.9968 \\
0.9822 \\
0.9685 \\
0.9685 \\
0.9685 \\
\end{tabular} & \begin{tabular}{|l|}
0.9968 \\
0.9896 \\
0.9424 \\
0.8978 \\
0.8978 \\
0.8978 \\
\end{tabular} & $\begin{array}{l}0.9822 \\
0.9424 \\
0.6820 \\
0.4361 \\
0.4361 \\
0.4361\end{array}$ & \begin{tabular}{|c|}
0.9685 \\
0.8978 \\
0.4361 \\
0.0001 \\
0.0 \\
0.0 \\
\end{tabular} & \begin{tabular}{|c|}
0.9685 \\
0.8978 \\
0.4361 \\
0.0 \\
0.0 \\
0.0 \\
\end{tabular} & \begin{tabular}{|c|}
0.9685 \\
0.8978 \\
0.4361 \\
0.0 \\
0.0 \\
0.0 \\
\end{tabular} \\
\hline FINITE DIFFERENCE & $\begin{array}{l}0.0025 \\
0.0081 \\
0.0493 \\
0.2575 \\
1.3117 \\
6.6485\end{array}$ & $\begin{array}{l}0.9990 \\
0.9967 \\
0.9818 \\
0.9681 \\
0.9681 \\
0.9681\end{array}$ & $\begin{array}{l}0.9967 \\
0.9893 \\
0.9408 \\
0.8966 \\
0.8966 \\
0.8966\end{array}$ & $\begin{array}{l}0.9818 \\
0.9408 \\
0.6724 \\
0.4275 \\
0.4273 \\
0.4273\end{array}$ & $\begin{array}{l}0.9682 \\
0.8967 \\
0.4281 \\
0.0007 \\
0.0004 \\
0.0004\end{array}$ & $\begin{array}{c}0.9681 \\
0.8967 \\
0.4279 \\
0.0004 \\
0.0 \\
0.0\end{array}$ & $\begin{array}{c}0.9681 \\
0.8967 \\
0.4279 \\
0.0004 \\
0.0 \\
0.0\end{array}$ \\
\hline SPLINE 2 & $\begin{array}{l}0.0025 \\
0.0081 \\
0.0493 \\
0.2575 \\
1.3117 \\
6.6485\end{array}$ & $\begin{array}{l}0.9990 \\
0.9968 \\
0.9821 \\
0.9683 \\
0.9683 \\
0.9683\end{array}$ & $\begin{array}{l}0.9968 \\
0.9895 \\
0.9418 \\
0.8973 \\
0.8973 \\
0.8973\end{array}$ & $\begin{array}{l}0.9821 \\
0.9419 \\
0.6790 \\
0.4330 \\
0.4332 \\
0.4332\end{array}$ & $\begin{array}{l}0.9684 \\
0.8974 \\
0.4336 \\
-.0005 \\
-.0003 \\
-.0003\end{array}$ & $\begin{array}{c}0.9684 \\
0.8974 \\
0.4338 \\
-.0003 \\
0.0 \\
0.0\end{array}$ & $\begin{array}{c}0.9684 \\
0.8974 \\
0.4338 \\
-.0003 \\
0.0 \\
0.0\end{array}$ \\
\hline SPLINE 4 & $\begin{array}{l}0.0025 \\
0.0081 \\
0.0493 \\
0.2575 \\
1.3117 \\
6.6485\end{array}$ & $\begin{array}{l}0.9990 \\
0.9968 \\
0.9822 \\
0.9865 \\
0.9684 \\
0.9684\end{array}$ & $\begin{array}{l}0.9968 \\
0.9895 \\
0.9423 \\
0.8977 \\
0.8977 \\
0.8977\end{array}$ & $\begin{array}{l}0.9822 \\
0.9424 \\
0.6820 \\
0.4360 \\
0.4358 \\
0.4358\end{array}$ & $\begin{array}{l}0.9685 \\
0.8979 \\
0.4366 \\
0.0007 \\
0.0003 \\
0.0003\end{array}$ & $\begin{array}{c}0.9685 \\
0.8978 \\
0.4364 \\
0.0003 \\
0.0 \\
0.0\end{array}$ & $\begin{array}{c}0.9685 \\
0.8978 \\
0.4364 \\
0.0003 \\
0.0 \\
0.0\end{array}$ \\
\hline
\end{tabular}




\section{TABLE 8 \\ SOLUTION OF THE LAPLACE EQUATION}

\begin{tabular}{|l|l|l|l|l|l|l|}
\hline METHOD & $-u(r, 0)$ & $u\left(.5, h_{2}\right)$ & $\sigma$ & $k_{2}$ & $h_{2}$ & $Y_{\text {MAX }}$ \\
\hline EXACT SOLUTION & 3.142 & 0.7304 & 1.0 & 0.1 & 0.1 & 1.0 \\
FINITE DIFFERENCE & 3.123 & 0.7322 & & & & \\
SPLINE 2 & 3.164 & 0.7286 & & & & \\
SPLINE 4X, SPLINE 2Y & 3.154 & 0.7295 & & & & \\
SPLINE 4 & 3.142 & 0.7304 & & & & \\
\hline EXACT SOLUTION & 3.142 & 0.5335 & 1.0 & 0.1 & 0.2 & 2.0 \\
SPLINE 4 & 3.162 & 0.5329 & & & & \\
SPLINE 4X, SPLINE 2Y & 3.193 & 0.5277 & & & & \\
FINITE DIFFERENCE & $2.828 *$ & 0.5401 & & & & \\
EXACT SOLUTION & 3.142 & 0.9691 & 1.7 & 0.1 & 0.01 & 2.86 \\
FINITE DIFFERENCE & 3.189 & 0.9686 & & & & \\
SPLINE 2 & 3.175 & 0.9687 & & & & \\
SPLINE 4X, SPLINE 2Y & 3.162 & 0.9689 & & & & \\
SPLINE 4 & 3.137 & 0.9691 & & & & \\
\hline EXACT SOLUTION & 3.142 & 0.9391 & 1.7 & 0.1 & 0.02 & 2.73 \\
FINITE DIFFERENCE & 3.193 & 0.9381 & & & & \\
SPLINE 2 & 3.177 & 0.9384 & & & & \\
SPLINE 4X, SPLINE 2Y & 3.164 & 0.9387 & & & & \\
EXACT SOLUTION & 3.142 & 0.7304 & 1.7 & 0.1 & 0.1 & 28.66 \\
FINITE DIFFERENCE & 3.220 & 0.7233 & & & & \\
SPLINE 4X, SPLINE 2Y & 3.185 & 0.7263 & & & & \\
SPLINE 4 & 3.130 & 0.7313 & & & & \\
\hline EXACT SOLUTION & 3.1416 & 0.9691 & $17 / .2$ & 0.1 & 0.01 & 1.063 \\
FINITE DIFFERENCE & 3.1694 & 0.9688 & & & & \\
SPLINE 2 & 3.1676 & 0.9688 & & & & \\
SPLINE 4X, SPLINE 2Y & 3.1551 & 0.9689 & & & & \\
SPLINE 4 & 3.1404 & 0.9691 & & & & \\
\hline
\end{tabular}

* eVAluated by 3-POINT end-DIfFerence formula 
TABLE 9

POTENTIAL FLOW OVER A CIRCULAR CYLINDER

\begin{tabular}{|c|c|c|c|c|c|c|}
\hline METHOD & $r \theta$ & 0 & $\pi / 10$ & $2 \pi / 10$ & $3 \pi / 10$ & $4 \pi / 10$ \\
\hline $\begin{array}{l}\text { EXACT SOLUTION } \\
\Delta \theta=\pi / 10 \\
h_{2}=0.05 \\
\sigma=1.7 \\
r_{\text {MAX }}=15.3285\end{array}$ & \begin{tabular}{|l|}
1.0500 \\
1.2795 \\
1.9428 \\
3.8596 \\
9.3991
\end{tabular} & $\begin{array}{l}2.0024 \\
2.0611 \\
2.4575 \\
4.1187 \\
9.5055\end{array}$ & \begin{tabular}{|l|}
1.9044 \\
1.9602 \\
2.3372 \\
3.9171 \\
9.0403 \\
\end{tabular} & $\begin{array}{l}1.6200 \\
1.6674 \\
1.9881 \\
3.3321 \\
7.6901\end{array}$ & \begin{tabular}{|l|}
1.1770 \\
1.2115 \\
1.4445 \\
2.4209 \\
5.5872 \\
\end{tabular} & $\begin{array}{l}0.6188 \\
0.6369 \\
0.7594 \\
1.2727 \\
2.9374\end{array}$ \\
\hline FINITE DIFFERENCE & $\begin{array}{l}1.0500 \\
1.2795 \\
1.9428 \\
3.8596 \\
9.3991\end{array}$ & $\begin{array}{l}1.8626 \\
1.9365 \\
2.3597 \\
4.0573 \\
9.4618\end{array}$ & \begin{tabular}{|l|}
1.7714 \\
1.8417 \\
2.2442 \\
3.8587 \\
8.9987
\end{tabular} & $\begin{array}{l}1.5069 \\
1.5667 \\
1.9091 \\
3.2824 \\
7.6547\end{array}$ & \begin{tabular}{|l|}
1.0948 \\
1.1383 \\
1.3871 \\
2.3848 \\
5.5615
\end{tabular} & $\begin{array}{l}0.5757 \\
0.5985 \\
0.7293 \\
1.2538 \\
2.9239\end{array}$ \\
\hline SPLINE 2 & $\begin{array}{l}1.0500 \\
1.2795 \\
1.9428 \\
3.8596 \\
9.3991\end{array}$ & $\begin{array}{l}1.9249 \\
1.9821 \\
2.3726 \\
4.0285 \\
9.4254\end{array}$ & $\begin{array}{l}1.8307 \\
1.8851 \\
2.2565 \\
3.8314 \\
8.9641\end{array}$ & $\begin{array}{l}1.5573 \\
1.6035 \\
1.9195 \\
3.2591 \\
7.6253\end{array}$ & $\begin{array}{l}1.1314 \\
1.1650 \\
1.3946 \\
2.3679 \\
5.5401\end{array}$ & $\begin{array}{l}0.5948 \\
0.6125 \\
0.7332 \\
1.2449 \\
2.9126\end{array}$ \\
\hline SPLINE 4 & $\begin{array}{l}1.0500 \\
1.2795 \\
1.9428 \\
3.8596 \\
9.3991\end{array}$ & $\begin{array}{l}2.0089 \\
2.0677 \\
2.4639 \\
4.1185 \\
9.4760\end{array}$ & $\begin{array}{l}1.9106 \\
1.9665 \\
2.3433 \\
3.9169 \\
9.0122\end{array}$ & $\begin{array}{l}1.6253 \\
1.6728 \\
1.9934 \\
3.3319 \\
7.6663\end{array}$ & $\begin{array}{l}1.1808 \\
1.2154 \\
1.4483 \\
2.4208 \\
5.5699\end{array}$ & $\begin{array}{l}0.6208 \\
0.6390 \\
0.7614 \\
1.2727 \\
2.9283\end{array}$ \\
\hline
\end{tabular}


TABLE 10

POTENTIAL FLOW OVER A CIRCULAR CYLINDER

\begin{tabular}{|c|c|c|c|c|c|c|}
\hline METHOD & $r \theta$ & 0 & $\pi / 10$ & $2 \pi / 10$ & $3 \pi / 10$ & $4 \pi / 10$ \\
\hline \multirow{3}{*}{$\begin{array}{l}\text { EXACT SOLUTION } \\
\Delta \theta=\pi / 20 \\
h_{2}=0.05 \\
\sigma=1.7 \\
r_{\text {MAX }}=15.3285\end{array}$} & $\begin{array}{l}1.0500 \\
1.2795\end{array}$ & $\begin{array}{l}2.0024 \\
2.0611\end{array}$ & $\begin{array}{l}1.9044 \\
1.9602\end{array}$ & $\begin{array}{l}1.6200 \\
1.6674\end{array}$ & $\begin{array}{l}1.1770 \\
1.2115\end{array}$ & $\begin{array}{l}0.6188 \\
0.6369\end{array}$ \\
\hline & 1.9428 & 2.4575 & 2.3372 & $\begin{array}{l}1.9881 \\
32301\end{array}$ & 1.4445 & $\begin{array}{l}0.7594 \\
1.2707\end{array}$ \\
\hline & $\begin{array}{l}3.8596 \\
9.3991\end{array}$ & $\begin{array}{l}4.1187 \\
9.5055\end{array}$ & $\begin{array}{l}3.9171 \\
9.0403\end{array}$ & $\begin{array}{l}3.3321 \\
7.6901\end{array}$ & $\begin{array}{l}2.4209 \\
5.5872\end{array}$ & $\begin{array}{l}1.2727 \\
2.9374\end{array}$ \\
\hline \multirow[t]{3}{*}{ FINITE DIFFERENCE } & 1.0500 & 1.9246 & 1.8304 & $1.5571^{\circ}$ & 1.1313 & 0.5949 \\
\hline & $\begin{array}{l}1.2795 \\
1.9428\end{array}$ & $\begin{array}{l}1.9830 \\
2.3816\end{array}$ & $\begin{array}{l}1.8859 \\
2.2650\end{array}$ & $\begin{array}{l}1.6043 \\
1.9268\end{array}$ & $\begin{array}{l}1.1657 \\
1.4000\end{array}$ & $\begin{array}{l}0.6130 \\
0.7361\end{array}$ \\
\hline & $\begin{array}{l}3.8596 \\
9.3991\end{array}$ & $\begin{array}{l}4.0551 \\
9.4506\end{array}$ & $\begin{array}{l}3.8566 \\
8.9881\end{array}$ & $\begin{array}{l}3.2807 \\
7.6457\end{array}$ & $\begin{array}{l}2.3836 \\
5.5549\end{array}$ & $\begin{array}{l}1.2532 \\
2.9204\end{array}$ \\
\hline SPLINE 2 & $\begin{array}{l}1.0500 \\
1.2795 \\
1.9428 \\
3.8596 \\
9.3991\end{array}$ & $\begin{array}{l}1.9413 \\
1.9986 \\
2.3898 \\
4.0474 \\
9.4399\end{array}$ & $\begin{array}{l}1.8463 \\
1.9008 \\
2.2728 \\
3.8494 \\
8.9778\end{array}$ & $\begin{array}{l}1.5706 \\
1.6169 \\
1.9334 \\
3.2745 \\
7.6370\end{array}$ & $\begin{array}{l}1.1412 \\
1.1749 \\
1.4048 \\
2.3791 \\
5.5486\end{array}$ & $\begin{array}{l}0.6001 \\
0.6178 \\
0.7387 \\
1.2509 \\
2.9171\end{array}$ \\
\hline SPLINE 4 & $\begin{array}{l}1.0500 \\
1.2795 \\
1.9428 \\
3.8596 \\
9.3991\end{array}$ & $\begin{array}{l}2.0094 \\
2.0682 \\
2.4643 \\
4.1188 \\
9.4762\end{array}$ & $\begin{array}{l}1.9111 \\
1.9670 \\
2.3437 \\
3.9172 \\
9.0124\end{array}$ & $\begin{array}{l}1.6257 \\
1.6732 \\
1.9938 \\
3.3323 \\
7.6664\end{array}$ & $\begin{array}{l}1.1813 \\
1.2158 \\
1.4487 \\
2.4211 \\
5.5700\end{array}$ & $\begin{array}{l}0.6212 \\
0.6394 \\
0.7618 \\
1.2730 \\
2.9284\end{array}$ \\
\hline
\end{tabular}




\section{TABLE ॥}

SLIP VELOCITY ON

THE FRONT OF A

CIRCULAR CYLINDER

$$
\Delta \theta=\pi / 10
$$

\begin{tabular}{|c|c|c|c|c|}
\hline$\theta$ & F. D. & SPLINE 2 & SPLINE 4 & EXACT \\
\hline$\pi / 10$ & -0.564 & -0.594 & -0.620 & -0.628 \\
$2 \pi / 10$ & -1.073 & -1.130 & -1.179 & -1.176 \\
$3 \pi / 10$ & -1.477 & -1.555 & -1.623 & -1.618 \\
$4 \pi / 10$ & -1.736 & -1.828 & -1.908 & -1.902 \\
$5 \pi / 10$ & -1.825 & -1.922 & -2.006 & -2.000 \\
\hline
\end{tabular}


TABLE 12

BLASIUS PROFILE: $\sigma=1.0, h_{2}=0.1, N=61$

\begin{tabular}{|c|c|c|c|c|c|c|c|c|}
\hline \multirow[b]{2}{*}{$x$} & & \\
\hline & F. D. & SPLINE 2 & SPLINE 4 & EXACT & F. D. & SPLINE 2 & SPLINE 4 & EXACT \\
\hline 1 & 0.002348 & 30.002348 & 0.002348 & 0.002348 & .046967 & 0.046962 & 959 & $0.04695 \mathrm{~s}$ \\
\hline & 0.009392 & 0.00 & 0.0 & 0.0 & 0.093923 & & & \\
\hline & 0.03 & & & & & & & \\
\hline & $0.0 \varepsilon$ & & & & & & & \\
\hline & 0.233026 & 0.232982 & 0.23 & 0.23 & 0.460788 & & 0.4 & 0.46 \\
\hline 1.5 & 0.515111 & 0.514990 & 0.51 & 0.5 & .661735 & 1379 & 174 & 0.66 \\
\hline & 0.886 & 30.88 & 0.8 & 0.88 & 0.817023 & 0.816600 & 995 & 0.8 \\
\hline & 2.78 & & & 2.783885 & 99782 & 0.99775 & 99777 & 99777 \\
\hline & & & & 4.783217 & & 1.0 & 1.0 & \\
\hline
\end{tabular}


TABLE 13

BLASIUS PROFILE: $\sigma=1.0, h_{2}=1.0, N=21$

\begin{tabular}{|c|c|l|l|l|l|l|l|l|}
\hline & \multicolumn{3}{|c|}{$f$} & \multicolumn{4}{c|}{$f^{\prime}$} \\
\cline { 2 - 9 } & F. D. & SPLINE 2 & SPLINE 4 & EXACT & F.D. & SPLINE 2 & SPLINE 4 & EXACT \\
\hline 1.0 & 0.23859 & 0.23768 & 0.23490 & 0.23299 & 0.47718 & 0.45853 & 0.46175 & 0.46063 \\
2.0 & 0.90351 & 0.89181 & 0.88831 & 0.88679 & 0.85265 & 0.81125 & 0.81795 & 0.81669 \\
3.0 & 1.82705 & 1.79403 & 1.80274 & 1.79557 & 0.99444 & 0.97059 & 0.96996 & 0.96905 \\
4.0 & 2.82470 & 2.77982 & 2.78658 & 2.78389 & 1.00085 & 0.99948 & 0.99701 & 0.99777 \\
5.0 & 3.82500 & 3.77942 & 3.78874 & 3.78323 & 0.99975 & 0.99999 & 1.00001 & 0.99994 \\
6.0 & 4.82492 & 4.77947 & 4.78555 & 4.78322 & 1.00010 & 1.00002 & 0.99989 & 1.0 \\
20.0 & 18.8249 & 18.7795 & 18.7857 & 18.78322 & 1.0 & 1.0 & 1.0 & 1.0 \\
\hline
\end{tabular}


TABLE 14

BLASIUS PROFILE: $\sigma=1.8 / 1, h_{2}=0.5, \mathrm{~N}=21$

\begin{tabular}{|c|c|l|l|l|l|l|l|l|}
\hline \multirow{2}{*}{$X$} & \multicolumn{4}{|c}{$f$} & \multicolumn{4}{c|}{$f^{\prime}$} \\
\cline { 2 - 9 } & F. D. & SPLINE 2 & SPLINE 4 & EXACT & F. D. & SPLINE 2 & SPLINE 4 & EXACT \\
\hline 0.5 & 0.05910 & 0.05835 & 0.05888 & 0.05864 & 0.23642 & 0.23230 & 0.23477 & 0.23423 \\
1.4 & 0.45557 & 0.45051 & 0.45722 & 0.45072 & 0.64461 & 0.61651 & 0.62568 & 0.62439 \\
2.4 & 1.24551 & 1.22531 & 1.24067 & 1.23153 & 0.93527 & 0.89654 & 0.90300 & 0.90107 \\
3.4 & 2.21456 & 2.17478 & 2.19064 & 2.18747 & 1.00284 & 0.99037 & 0.98802 & 0.98797 \\
4.4 & 3.21568 & 3.16972 & 3.18422 & 3.18338 & 0.99940 & 1.00010 & 0.99898 & 0.99940 \\
5.4 & 4.21548 & 4.16985 & 4.18386 & 4.18322 & 1.00020 & 1.00001 & 1.00003 & 0.99999 \\
19.4 & 18.2155 & 18.1698 & 18.18380 & 18.18322 & 1.0 & 1.0 & 1.0 & 1.0 \\
\hline
\end{tabular}




\section{TABLE $15 \quad f^{\prime \prime}(0)$ FOR BLASIUS EQUATION}

\begin{tabular}{|c|c|c|c|c|c|c|}
\hline$x_{\max }$ & $h_{2}$ & $\sigma$ & $\begin{array}{c}\text { FINITE } \\
\text { DIFFERENCE }\end{array}$ & SPLINE 2 & SPLINE 4 & $N_{6} / N$ \\
\hline 6.0 & 0.1 & 1.0 & 0.4697265 & 0.469634 & 0.469601 & $61 / 61$ \\
\hline 20.0 & 1.0 & 1.0 & 0.528041 & 0.475357 & 0.476359 & $7 / 21$ \\
\hline 5.6665 & 0.05 & 1.5 & 0.516646 & 0.470718 & 0.466048 & $11 / 11$ \\
\hline 11.3330 & 0.1 & 1.5 & 0.6049558 & $p$ & 0.493598 & $9 / 11$ \\
\hline 6.4344 & 0.2 & 1.5 & 0.498214 & $\rightleftharpoons$ & $=0.455623$ & $7 / 8$ \\
\hline 13.365 & 0.01 & $1.8 / 1$. & 0.474643 & & 0.469188 & $13 / 21$ \\
\hline 16.063 & 0.05 & $1.8 / 1$. & 0.473974 & & 0.46 & $10 / 21$ \\
\hline 19.400 & 0.5 & $1.8 / 1$. & 0.479715 & 0.466839 & 0.469509 & $7 / 21$ \\
\hline 37.020 & 0.5 & $1.8 / 2$ & 0.551803 & 0.460823 & 0.477930 & $5 / 21$ \\
\hline 53.936 & 0.5 & $1.8 / 3$ & 0.827648 & 0.506798 & 0.523256 & $5 / 21$ \\
\hline
\end{tabular}


TABLE 16 STAGNATION POINT FLOW

(a) $f^{\prime \prime}(0)$

\begin{tabular}{|c|c|c|c|c|c|c|}
\hline$x_{\text {max }}$ & $h_{2}$ & $\sigma$ & \begin{tabular}{|l|} 
FINITE \\
DIFFERENCE
\end{tabular} & SPLINE 2 & SPLINE 4 & $\mathrm{~N}_{5} / \mathrm{N}$ \\
\hline 6. & 0.1 & 1.0 & 1.23257 & 1.23227 & 1.23258 & $51 / 61$ \\
\hline 20.0 & 1.0 & 1.0 & 1.07167 & 1.20612 & 1.20882 & $6 / 21$ \\
\hline 4448 & .001 & $1.8 / 1$. & 1.26353 & 1.23604 & 1.23299 & | 16/21 \\
\hline 19.40 & 0.5 & $1.8 / 1$ & 1.24031 & 1.22764 & 1.23617 & 6/21 \\
\hline
\end{tabular}

$f^{\prime \prime}(0)=1.232588$ (ROSENHEAD ${ }^{(16)}$ )

(b) $f\left(h_{2}\right)$

\begin{tabular}{|r|c|c|c|c|c|c|}
\hline$x_{\max }$ & $h_{2}$ & $\sigma$ & $\begin{array}{c}\text { FINITE } \\
\text { DIFFERENCE }\end{array}$ & SPLINE 2 & SPLINE 4 & ROSENHEAD ${ }^{1161}$ \\
\hline 6.0 & 0.1 & 1.0 & 0.005915 & 0.005995 & 0.005996 & 0.005996 \\
20.0 & 1.0 & 1.0 & 0.390440 & 0.436393 & 0.450482 & 0.459227 \\
19.40 & 0.5 & $1.8 / 1$ & 0.128780 & 0.132622 & 0.135410 & 0.133585 \\
\hline
\end{tabular}




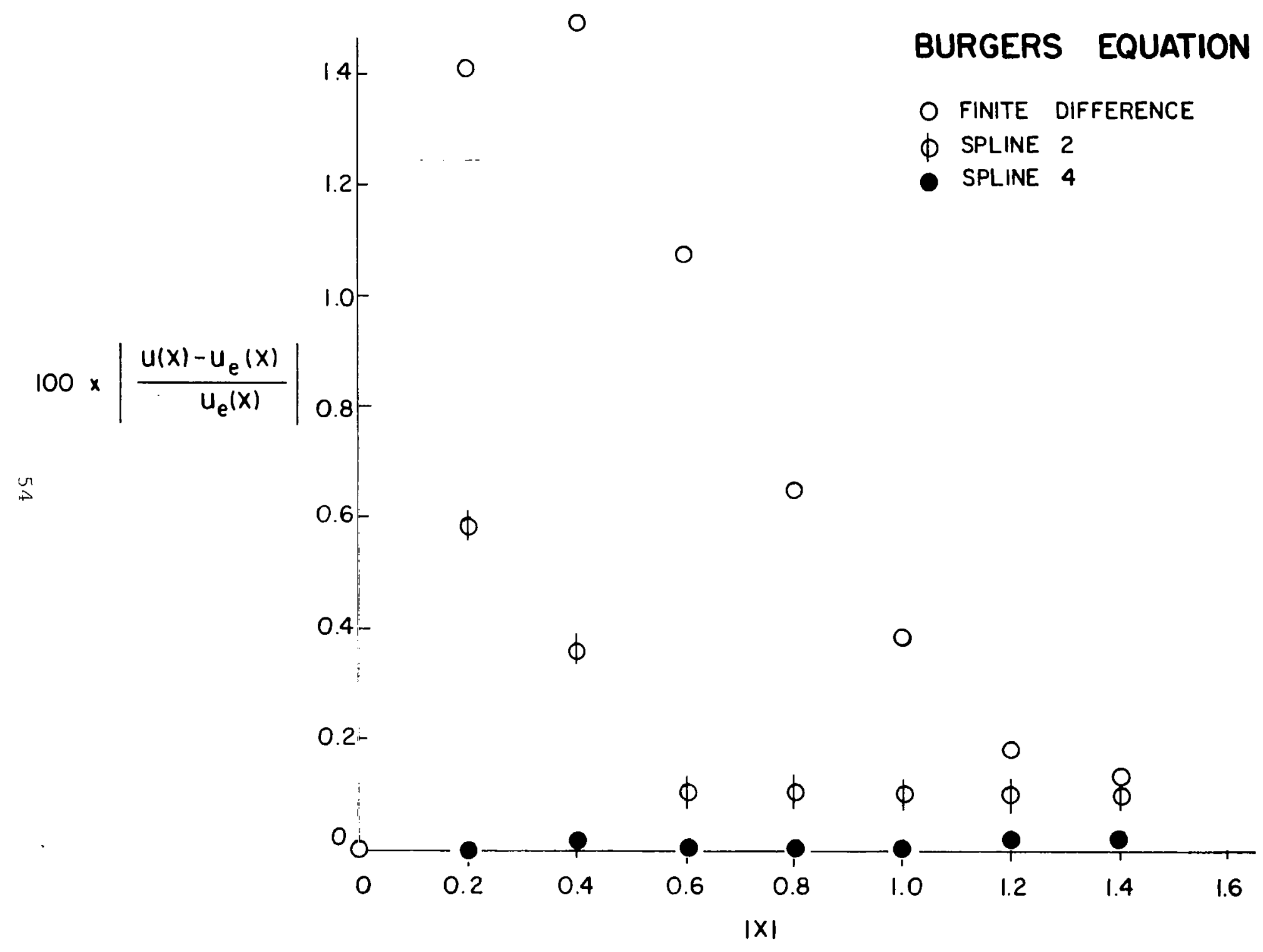

FIG. I NON-LINEAR BURGERS EQUATION: $\nu=1 / 8, \sigma=1$ 


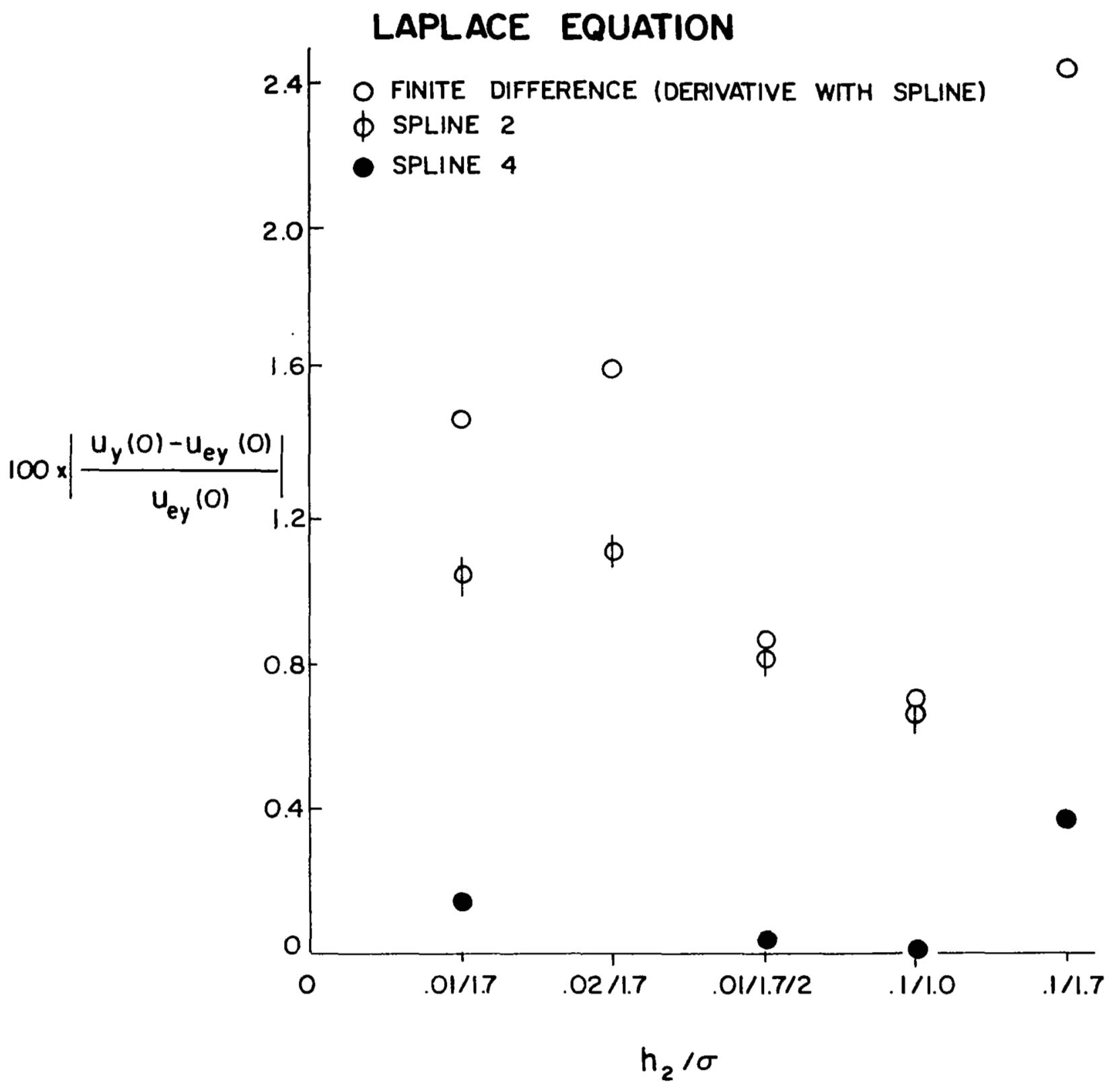

FIG. 2 LAPLACE EQUATION 


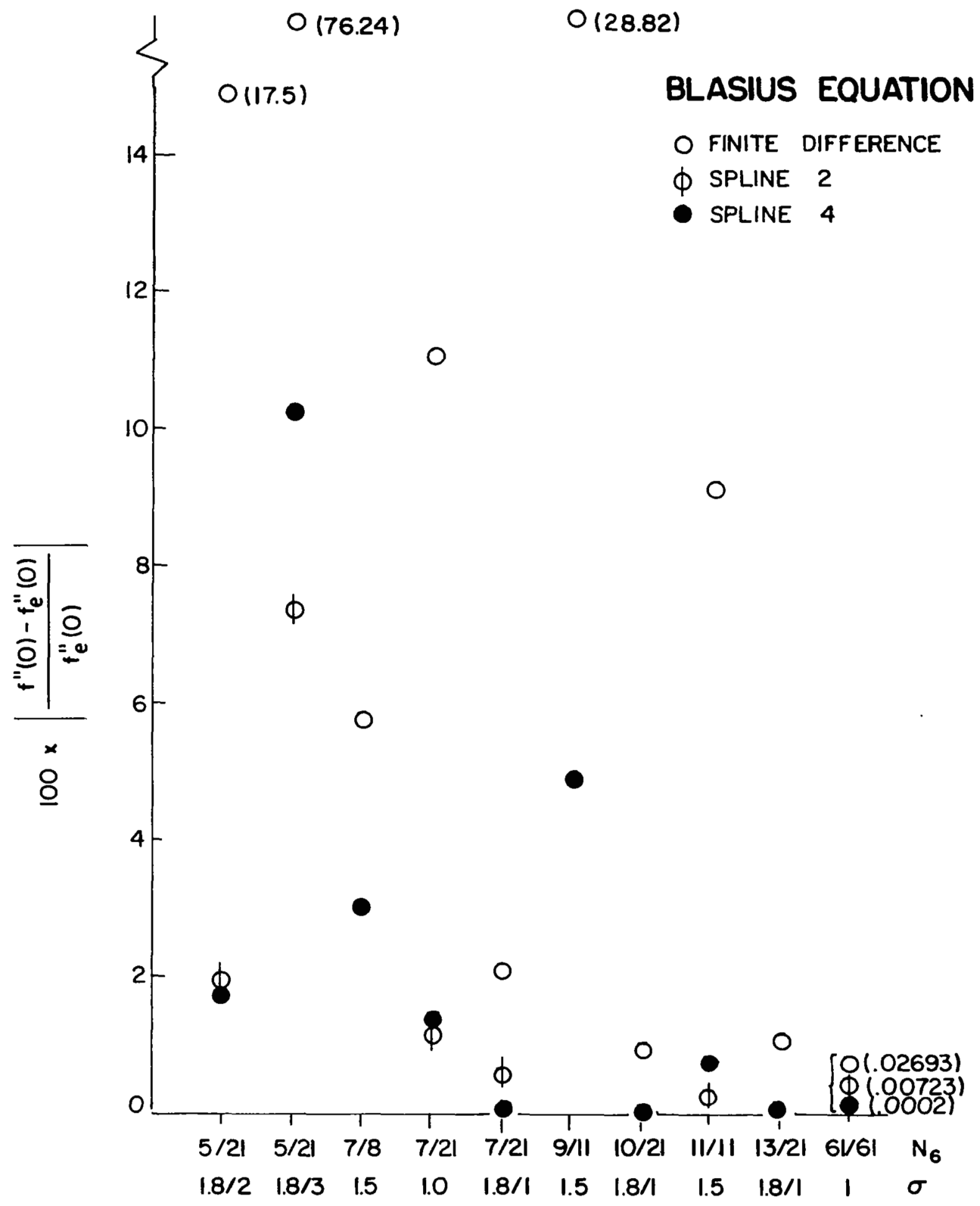

FIG. 3 ERROR PLOT: BLASIUS EQUATION 


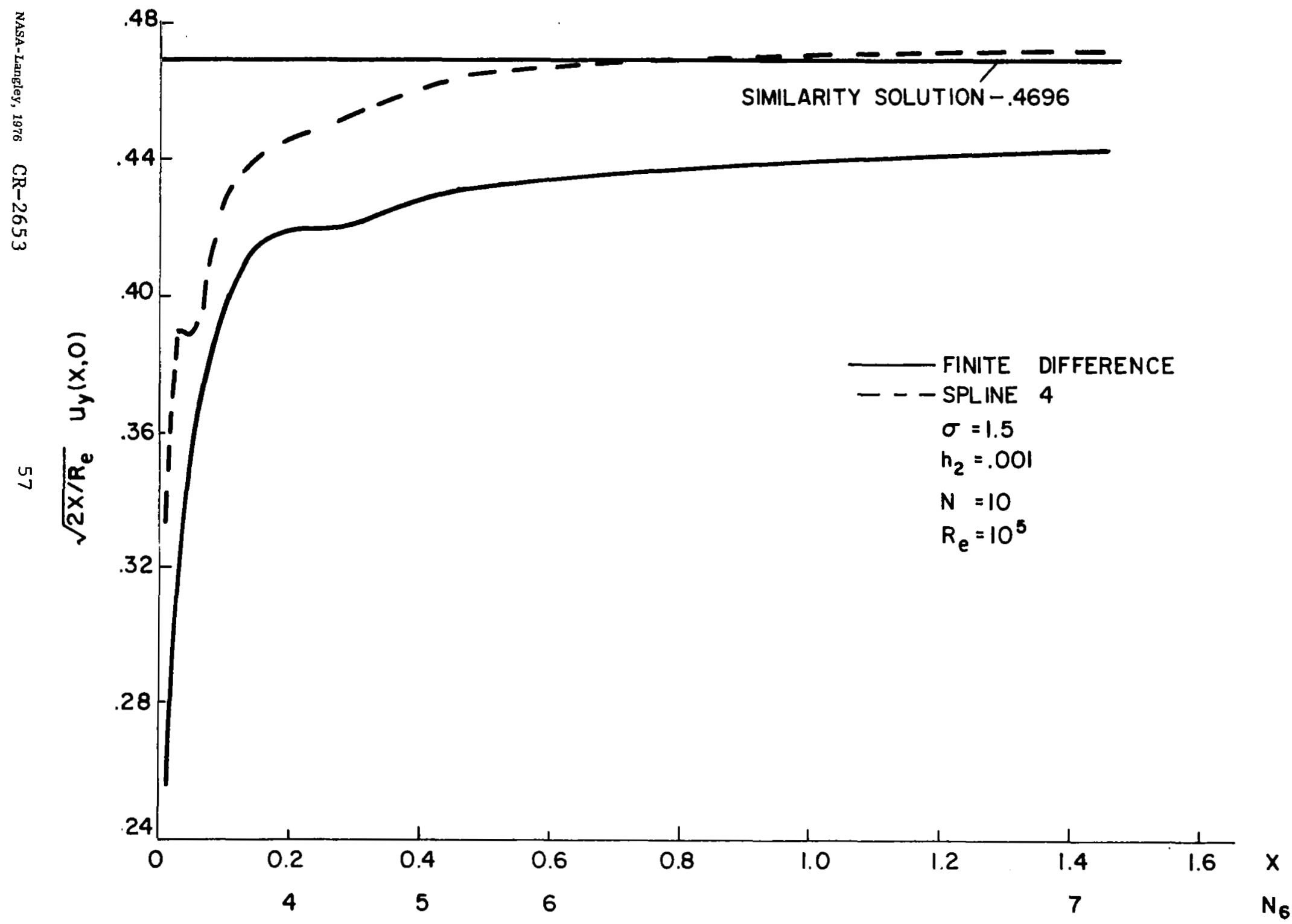

FIG.4 CONSTANT PRESSURE BOUNDARY LAYER SOLUTION-PHYSICAL VARIABLES 